\title{
Recommandations canadiennes pour les pratiques optimales de prise en charge de la sclérose latérale amyotrophique
}

\author{
Christen Shoesmith MD, Agessandro Abrahao MD MSc, Tim Benstead MD, Marvin Chum MD MSc, \\ Nicolas Dupre MD MSc, Aaron Izenberg MD, Wendy Johnston MD, Sanjay Kalra MD, Desmond Leddin MB MSc, \\ Colleen O’Connell MD, Kerri Schellenberg MD MÉdMéd, Anu Tandon MD, Lorne Zinman MD MSc
}

Citation : CMAJ 2020 November 16;192:E1453-68. doi : 10.1503/cmaj.191721-f

Balados du CMAJ : Entrevue (en anglais) avec les auteurs ici : www.cmaj.ca/lookup/doi/10.1503/cmaj.191721/tab-related-content

Voir la version anglaise de l'article ici : www.cmaj.ca/lookup/doi/10.1503/cmaj.191721

$\mathbf{L}$ a sclérose latérale amyotrophique (SLA) est une maladie progressive invalidante due à une dégénérescence des motoneurones dans le cerveau et la moelle épinière; elle provoque faiblesse, atrophie musculaire, fasciculations et spasticité . La forme la plus fréquente est une faiblesse qui affecte d'abord les membres, suivie de problèmes de mobilité chez près de $70 \%$ des patients ${ }^{2}$. La forme bulbaire, accompagnée d'une atteinte des muscles oropharyngés nuisant à la déglutition et au langage, s'observe dans environ $25 \%$ des cas $^{2}$. En plus de l'atteinte motrice, une dégénérescence au niveau des lobes frontaux et temporaux qui entraîne une atteinte cognitive ou comportementale, s'observe chez jusqu'à $50 \%$ des patients ${ }^{3}$. Avec le temps, la force diminue, et les patients décèdent en général d'insuffisance respiratoire dans les 5 ans qui suivent le diagnostic ${ }^{2}$. Malgré l'intensification de la recherche ces dernières années, les options thérapeutiques pour la SLA demeurent limitées et les soins aux patients concernent principalement le soulagement des symptômes et une optimisation du fonctionnement et de la qualité de vie².

On estime à 3000 le nombre de Canadiennes et de Canadiens actuellement aux prises avec la SLA ${ }^{4,5}$. Des groupes de défense des intérêts et des médecins qui soignent ces patients se sont mobilisés pour obtenir des recommandations sur les soins et la prise en charge optimale de ces patients au Canada. Des lignes directrices de pratique clinique sur la SLA ont été publiées aux États-Unis ${ }^{6,7}$ et en Europe ${ }^{8,9}$, mais à ce jour, aucune n'a porté spécifiquement sur les soins aux patients atteints de SLA au Canada.

En plus de fournir une mise à jour sur les normes thérapeutiques en constante évolution pour la SLA, les pratiques optimales recommandées dans cette ligne directrice permettent d'aborder plusieurs enjeux importants pour la population canadienne, tels que le soutien aux proches aidants, la gestion de la

\section{POINTS CLÉS}

- La prise en charge des patients atteints de sclérose latérale amyotrophique (SLA) requiert une approche holistique multidisciplinaire spécialisée.

- Les traitements pharmacologiques modificateurs de la maladie pour la SLA incluent le riluzole et l'édaravone.

- Il faut porter une grande attention au soutien nutritionnel et aux soins respiratoires pour une prise en charge optimale de la SLA.

- Plusieurs traitements sont disponibles pour alléger les symptômes de la SLA.

- Les soins palliatifs et le soutien aux proches aidants sont d'importants éléments pour accompagner les patients durant leur cheminement les confrontant à la SLA.

pharmacothérapie et l'aide médicale à mourir (AMM). La rédaction d'une première ligne directrice canadienne sur la SLA constitue une étape cruciale dans un processus qui permettra d'actualiser ces recommandations à mesure que de nouvelles données probantes se feront jour; sans compter qu'elle permettra d'identifier et de prioriser les sujets abordés par la recherche et de combler les lacunes sur le plan des connaissances.

Étant donné que la rigueur généralement attendue des recommandations médicales fondées sur des données probantes est difficile à observer pour ce qui est des différents aspects du traitement de la SLA, plusieurs des recommandations présentées ici reposent sur le consensus des experts relativement aux bonnes pratiques. En général, la prise en charge des symptômes de la SLA s'inspire des données probantes générées pour d'autres pathologies. Les recommandations de cette ligne directrice se fondent sur les meilleures données probantes disponibles et sur le consensus des experts quant aux pratiques 
optimales, et sont donc le reflet de l'expérience pratique des médecins canadiens qui soignent des patients atteints de la SLA. Cet article est un sommaire de la ligne directrice intégrale, que l'on peut consulter sur le site Web de la Société canadienne de la SLA, à l'adresse (www.als.ca/bpr-appendix).

\section{Portée}

Le but de cette ligne directrice est d'offrir aux médecins, aux autres professionnels de la santé et aux omnipraticiens qui soignent des personnes atteintes de la SLA des recommandations de pratiques optimales pour les soins et la prise en charge des patients vivant avec la SLA, sans égard aux questions de sexe, de genre, d'âge ou au stade de la maladie. Cette ligne directrice devrait mener à l'élaboration d'une norme nationale afin d'améliorer la qualité des soins, au bénéfice des patients, des familles et des proches aidants affectés par la SLA. Les groupes de défense (p. ex., la Société canadienne de la SLA et ses sections provinciales), les instances sanitaires, les gouvernements et les responsables des orientations politiques seront ainsi mieux à même d'établir des normes de soins et d'en faire la promotion.

\section{Recommandations}

Les soins et la prise en charge des patients atteints de SLA devraient toujours être centrés sur les patients, sans négliger les dimensions holistiques et émotionnelles de leur bien-être. C'est au patient qu'il revient de décider de son traitement, ce qui inclut l'option d'abaisser le niveau d'intervention.

Les recommandations pour la prise en charge des patients atteints de SLA au Canada se trouvent au tableau 1, regroupées par thème, avec le niveau des données probantes. Si les données probantes étaient insuffisantes ou absentes pour une question clé, nous avons formulé des recommandations fondées sur le consensus des experts après examen de la littérature disponible et de l'expérience clinique en matière de SLA, ou sur l'extrapolation des traitements prodigués pour d'autres maladies plus communes.

Les sections suivantes fournissent un contexte et expliquent certaines recommandations reposant sur des données probantes. Le tableau des données probantes, à l'annexe $C$ de la ligne directrice intégrale (accessible ici www.als.ca/bpr -appendix), fournit des détails sur les données probantes à l'appui de toutes les recommandations présentées au tableau 1. Pour une explication complète des données probantes à l'appui des recommandations, veuillez consulter l'annexe A (www.als.ca/bpr-appendix).

\section{Annonce du diagnostic}

La façon d'annoncer le diagnostic de SLA est source de mécontentement chez plusieurs patients et proches aidants ${ }^{10,11}$. Des recommandations générales ont été formulées sur la façon d'annoncer le diagnostic de SLA ${ }^{8}$. Les médecins devraient principalement s'adapter aux besoins de chaque patient. Si un patient est incapable d'absorber la nouvelle du diagnostic de SLA, il peut être préférable d'y aller par étapes, sans tout dévoiler à la fois ${ }^{12}$.
À l'inverse, d'autres patients peuvent avoir l'impression d'avoir trop peu d'informations lors du diagnostic; les patients et les proches aidants souhaitent parfois qu'on les renseigne sur l'état de la recherche, des traitements et du pronostic au moment du diagnostic ${ }^{13}$.

Dans une étude sur la satisfaction quant à la façon d'annoncer le diagnostic de SLA, $41 \%$ des patients ont indiqué avoir reçu des renseignements insuffisants, et un tiers a précisé ne pas avoir eu les coordonnées d'une personne-ressource auprès de qui faire un suivi ${ }^{10}$. En outre, environ $75 \%$ des patients et des proches aidants avaient des questions immédiatement après l'annonce du diagnostic ${ }^{11}$. Ces observations rappellent qu'après l'annonce du diagnostic, les médecins doivent proposer des sources d'information, fournir les coordonnées de ressources communautaires et offrir un suivi rapide. Les patients se disent plus satisfaits de l'annonce du diagnostic de SLA s'ils croient que le médecin a compris ce qu'ils ressentent ${ }^{13}$. Une source additionnelle de frustration pour les patients a été le retard de confirmation du diagnostic, y compris le temps d'attente pour voir un spécialiste de la $\mathrm{SLA}^{10}$. Le groupe de travail a fixé à 4 semaines le délai maximal raisonnable pour obtenir une consultation de confirmation d'un diagnostic de SLA.

\section{Traitement modificateur de la maladie}

En 2000, Santé Canada a approuvé le riluzole pour le traitement de la SLA. Selon une méta-analyse de classe I regroupant 4 essais randomisés et contrôlés (ERC), le riluzole offre un avantage modeste sur la survie comparativement au placebo, avec un rapport de risque (HR) de 0,84 (intervalle de confiance [IC] de 95\% 0,698 à 0,997), représentant un gain de $9 \%$ de la probabilité de survie annuelle. Cela se traduit par une augmentation médiane de la survie de 11,8 à 14,8 mois $^{14}$. De récentes études de cohorte (toutes de classe III) basées sur un registre ont estimé l'amélioration de la survie médiane avec le traitement par riluzole à 7,3 mois $^{15}, 10$ mois $^{16}$ ou 12 mois $^{17}$, tandis que d'autres études n'ont fait état d'aucun effet sur la survie ${ }^{18-21}$. Les résultats d'autres études de cohorte de classe III ont donné des estimations du HR de $0,34^{22}, 0,71^{23}, 0,79^{24}$ et $0,81^{25}$, ce qui se traduit par une augmentation absolue de la survie annuelle estimée allant de $10 \%$ à $50 \%$.

Aucun essai contrôlé n'a vérifié si le riluzole prolonge la vie à un stade spécifique ou à tous les stades de la SLA. Selon une analyse post hoc de l'étude originale d'établissement des doses, le riluzole pourrait être efficace à prolonger la survie uniquement aux stades plus avancés de la maladie (définis par une détérioration nutritionnelle ou respiratoire suffisante pour nécessiter une intervention ${ }^{26}$, mais les résultats d'autres études de cohorte diffèrent, montrant qu'il pourrait être efficace uniquement lors des stades moins avancés ${ }^{27}$ ou que son effet sur la survie est de courte durée ${ }^{28}$. Néanmoins, des décennies d'expérience à l'échelle mondiale ont montré que le riluzole est en général bien toléré lors d'une utilisation prolongée et moyennant une surveillance régulière des enzymes hépatiques et des numérations sanguines, des nausées et de la fatigue (classe I) ${ }^{14}$.

En octobre 2018, Santé Canada a approuvé l'édaravone pour traiter la SLA. Une seule étude de classe 1 dans une population généralisée souffrant de SLA a échoué à démontrer un bienfait 
général de l'édaravone sur le ralentissement de la progression du score ALSFRS-R (ALS functional rating scale - revised) sur une période de 6 mois $^{29}$, mais a évoqué un avantage chez un sousgroupe de patients (voir tableau 1 pour les caractéristiques de ce sous-groupe). Cet effet bénéfique sur le ralentissement de la progression du score ALSFRS-R a par la suite été confirmé par une deuxième étude de classe 1 qui restreignait le recrutement aux patients présentant les caractéristiques du sous-groupe de la première étude ${ }^{30}$. La seconde étude a démontré une réduction moyenne du changement du score ALSFRS-R sur une période de 6 mois de 2,49 (IC de $95 \%$ 0,99 à 3,98). Pour l'instant, les données probantes disponibles laissent entendre des données probantes de niveau $B$, « probablement efficace » chez un groupe sélectionné de patients atteints de SLA.

Tableau 1 (partie 1 de 6) : Recommandations assorties du niveau de données probantes pour la prise en charge des patients atteints de sclérose latérale amyotrophique*

\section{Recommandations}

\section{Annonce du diagnostic}

- L'annonce du diagnostic doit se faire selon une approche adaptée aux besoins individuels du patient (CE).

- Le diagnostic de SLA doit être confirmé par un neurologue ou un physiatre qui connaît bien la SLA (CE). Les patients faisant l'objet d'une demande de confirmation d'un diagnostic initial de SLA devraient être vus dans une clinique de SLA dans les 4 semaines suivantes (CE). On recommande une prise de contact rapide par la clinique de SLA une fois le diagnostic confirmé (CE).

- Il faut discuter des traitements et de la recherche sur la SLA, remettre aux patients de la documentation écrite (imprimée ou électronique) sur les ressources pour la SLA et les encourager à devenir membres de leur association locale et nationale de la SLA (CE).

- Il faut éventuellement parler du pronostic en tenant compte de l'état de préparation du patient, sans l'aborder nécessairement dès l'annonce initiale (à moins que le patient n'en fasse la demande spécifiquement) (CE).

\section{Traitement modificateur de la maladie}

- Les traitements modificateurs de la maladie doivent être prescrits par des médecins qui connaissent bien la prise en charge de la SLA (CE).

- Riluzole :

- Le riluzole s'est révélé efficace pour améliorer la survie des patients atteints de la SLA (niveau A).

- Des données probantes montrent que le riluzole prolonge la survie pendant une durée médiane de 3 mois (niveau A).

- Il faut commencer le riluzole peu après le diagnostic de SLA (CE).

- Il est important d'exercer une surveillance régulière des effets indésirables possibles du riluzole (CE).

- Les données sont insuffisantes pour suggérer un épuisement de l'effet du riluzole à mesure que la maladie progresse, y compris l'insuffisance respiratoire (CE).

- Édaravone :

- Chez certains patients, l'édaravone intraveineuse a permis de ralentir le déclin des scores ALSFRS-R comparativement au placebo intraveineux, sur une période de 6 mois *niveau B). (Ces patients ont retiré des bienfaits de l'édaravone : durée de la maladie $<2$ ans, CVF $>80 \%$, tous les éléments du score ALSFRS-R $>2$ et déclin constant du score ALSFRS-R sur un intervalle de 3 mois.)

- Aucun signe de bienfaits de l'édaravone intraveineuse à d'autres stades de la SLA n'a été démontré (CE).

- Comme avec d'autres traitements, il faut soupeser soigneusement les objectifs, les risques et les avantages individuels et en discuter avant d'instaurer un traitement intraveineux par édaravone (CE).

- On encourage les médecins à parler ouvertement avec leurs patients des risques et avantages potentiels des traitements non officiellement approuvés (CE).

\section{Soins multidisciplinaires}

- Il faut orienter les patients atteints de SLA vers des cliniques multidisciplinaires spécialisées pour des soins optimaux (niveau B).

- Il faut expliquer aux patients et aux autorités médicales pourquoi le patient devrait être suivi dans une clinique multidisciplinaire. En voici les avantages:

- Avantage sur la survie (niveau B)

- Hospitalisations plus brèves et moins fréquentes que chez les patients qui ne sont pas suivis dans ces cliniques (niveau B)

- Utilisation accrue d'équipement adapté (niveau C)

- Utilisation accrue du riluzole, des sondes d'alimentation percutanées et de la VNE (niveau B)

- Amélioration de la QdV (niveau C)

- Les soins multidisciplinaires doivent être offerts par une équipe composée de médecins et d'autres professionnels de la santé qui s'occuperont de certains aspects comme la communication, la nutrition, la déglutition, la mobilité, les activités de la vie quotidienne, les soins respiratoires, la capacité cognitive, les enjeux psychosociaux, la prise en charge médicale et les soins de fin de vie (CE).

- La fréquence des visites à la clinique multidisciplinaire dépendra des besoins du patient et de la vitesse de progression de son état (CE).

- Une infirmière pivot ou autre professionnel de la santé de la clinique devrait être affectée au patient et à ses proches comme personne-ressource pour toutes les questions relatives à la SLA entre leurs visites à la clinique (CE).

- Un suivi par télémédecine et des soins virtuels sont envisageables et pourraient compléter les soins multidisciplinaires fournis par la clinique (niveau C). 
Tableau 1 (partie 2 de 6): Recommandations assorties du niveau de données probantes pour la prise en charge des patients atteints de sclérose latérale amyotrophique*

\section{Recommandations}

\section{Prise en charge respiratoire}

Dépistage

- Les patients atteints de SLA ont besoin d'une surveillance respiratoire régulière, au départ puis tous les 3 mois, ou selon l'indication clinique (CE). La surveillance respiratoire régulière devrait inclure :

- Revue des symptômes, tels que dyspnée, orthopnée et céphalées matinales (niveau C)

- Mesure de la CVF ou de la CVL en position assise (niveau B)

- Un ou plus des éléments suivants : PIN, CVF ou PIM en position couchée (niveau C)

- Mesure des gaz artériels, des gaz veineux ou du $\mathrm{CO}_{2}$ transcutané, lorsqu'on soupçonne une hypercapnie ou lorsque le syndrome bulbaire empêche des tests précis (niveau C)

- Mesure du DPT pour évaluer l'efficacité de la toux (niveau C)

- Oxymétrie ou polysomnographie nocturnes, quand on soupçonne des difficultés respiratoires symptomatiques durant le sommeil et en l'absence de critères diurnes pour l'instauration de la VNE (niveau C)

Ventilation

- La VNE est la norme de soins en cas d'insuffisance respiratoire dans la SLA, tant pour prolonger la survie que pour soulager les symptômes (niveau B).

- Les critères pour l'instauration de la VNE sont l'un ou l'autre des éléments suivants :

- Symptômes d'insuffisance respiratoire, y compris orthopnée (niveau B)

- $\mathrm{PIN} \leq 40 \mathrm{~cm} \mathrm{H} \mathrm{O}_{2}$ ou PIM $\leq 40 \mathrm{~cm} \mathrm{H}_{2} \mathrm{O}$ (niveau C)

- CVF fiable en position redressée $†<5 \%$ (CE)

- CVF en position assise ou couchée $<80 \%$ et signes ou symptômes d'insuffisance respiratoire (niveau B)

- Hypercapnie diurne $\mathrm{pCO}_{2}>45 \mathrm{~mm} \mathrm{Hg}$ (niveau B)

- Oxymétrie nocturne anormale ou difficultés respiratoires liées au sommeil (niveau B)

- Un spécialiste de la respiration doit être consulté pour instaurer la VNE (CE).

- Chez les patients qui présentent les critères ci-dessus, la VNE doit être instaurée dans les 4 semaines, mais les patients gravement symptomatiques auront besoin qu'on l'instaure plus rapidement. La polysomnographie nocturne n'est pas requise pour instaurer la VNE (CE).

- Veiller à un soutien à domicile pour la VNEł à des fins d'éducation, de titrage et de résolution de problèmes (CE).

- Les patients doivent être informés que le recours à la VNE pourrait changer leur trajectoire de survie avec la SLA et leur expérience de fin de vie (EC).

- La VNE améliore la QdV des patients atteints de SLA qui souffrent d'insuffisance respiratoire (niveau B).

- Il devrait y avoir des évaluations régulières en inhalothérapie spécialisée pour optimiser les modes, les pressions et les interfaces de la VNE. La surveillance doit inclure le téléchargement des données de l'appareil et peut inclure l'oxymétrie nocturne (niveau C).

- Il ne faut pas considérer l'oxygénothérapie comme un traitement de routine pour l'insuffisance respiratoire chronique. Chez les patients atteints de SLA qui souffrent d'hypoxémie aiguë, la gestion de l'insuffisance respiratoire par VNE doit être envisagée d'abord. Si l'hypoxémie persiste après après avoir atteint une pression VNE optimale, on évalue l'étiologie de l'hypoxémie et on peut envisager une oxygénothérapie d'appoint (CE).

- Il ne faut pas utiliser l'électro-entraînement diaphragmatique chez les patients atteints de la SLA parce qu'il est inefficace et peut nuire aux patients (niveau B).

- La VNE est une approche recommandée, même lorsque la ventilation est requise 24 heures sur 24 (CE).

- La ventilation par pièce buccale peut être envisagée chez certains patients comme forme de VNE durant le jour en plus de la VNE nocturne (CE).

- S'il est impossible de venir à bout efficacement de l'insuffisance respiratoire avec la VNE, une ventilation effractive est une option pour certains patients. Il faut avoir discuté des objectifs thérapeutiques et des directives préalables bien avant l'apparition de l'insuffisance respiratoire (CE).

- Les patients doivent comprendre que la SLA continuera de progresser malgré le soutien respiratoire (CE).

- Les discussions sur la planification préalable des soins doivent inclure des renseignements explicites sur toutes les interventions respiratoires. II faut mentionner que l'intubation pourrait être irréversible selon le stade de la maladie, et mentionner les options palliatives en cas d'essoufflement (voir la rubrique sur les soins palliatifs). Il faut aussi aborder l'option d'un arrêt de tout traitement en cours (CE).

- La trachéotomie est à envisager en cas d'obstruction des voies respiratoires supérieures avec paralysie des cordes vocales; toutefois, il faut aussi discuter de la ventilation effractive à long terme (CE).

Clairance des voies respiratoires

- Des stratégies de recrutement de volume pulmonaire (niveau C) et des techniques manuelles d'assistance à la toux (CE) doivent être instaurées lorsque les patients ont de la difficulté à éliminer leurs sécrétions.

- L'IEM biquotidienne est à envisager pour éliminer les sécrétions chez les patients atteints de SLA qui ont un DPT faible (<270 L/min). II faut augmenter la fréquence de l'IEM en cas d'infection respiratoire aiguë (CE).

- Assurer un soutien respiratoire à domicileł pour l'IEM à des fins d'éducation, de titrage et de résolution des problèmes (CE).

- La pharmacothérapie par mucolytiques (p. ex., guaïfénésine ou N-acétylcystéine), antagonistes des $\beta$ récepteurs (p. ex., métoprolol ou propranolol) ou solution saline ou ipratropium en nébulisation peuvent être envisagés (CE). 
Tableau 1 (partie 3 de 6): Recommandations assorties du niveau de données probantes pour la prise en charge des patients atteints de sclérose latérale amyotrophique*

\section{Recommandations}

\section{Nutrition}

Surveillance et intervention entérale

- Il faut surveiller l'état nutritionnel en mesurant le poids et l'IMC tous les 3 mois, ou selon l'indication clinique (niveau B); on peut tenir compte de la DÉQT (niveau B).

- Des interventions nutritionnelles (p. ex., modifications diététiques, pose d'une sonde entérale) sont envisageables au moment du diagnostic ou lors du suivi, en présence de : 1) risque accru d'aspiration malgré la modification de la consistance des aliments et recommandations compensatoires (CE); 2 ) réduction $\geq 5-10 \%$ du poids par rapport au poids habituel ou de référence (niveau C); 3 ) réduction $\geq 1$ point de l'IMC par rapport à l'IMC habituel ou de référence (niveau B); 4) IMC < 18,5 (niveau B); ou 5) DÉQT excédant l'apport énergétique quotidien (CE).

- L'information sur les avantages et risques des sondes d'alimentation entérale doit être fournie dès le début de la prise en charge de la SLA (CE).

- Quand la baisse de la CVF avoisine 50\%, il faut demander une consultation pour sonde entérale, même en l'absence de dysphagie. Une CVF <50\% n'empêche pas nécessairement la recommandation de la sonde d'alimentation entérale si l'état respiratoire est suivi étroitement durant et après l'intervention (niveau C). La VNE pourrait rendre la GEP ou la GRG plus sécuritaires chez les patients en insuffisance respiratoire (CE).

- Il faut faire vérifier la déglutition régulièrement par un professionnel de la santé certifié (p. ex. orthophoniste, ergothérapeute) (niveau B). Des mesures objectives de la déglutition (RBM ou EEFO-D) peuvent être utilisées initialement et durant la prise en charge de la SLA (CE).

Délai maximal avant une GEP ou une GRG

- Une fois que la décision de poser une sonde d'alimentation entérale est prise, l'insertion devrait se faire dans les 4 semaines. L'équipe de SLA doit avoir accès à des endoscopistes et à des radiologistes qui s'intéressent à cette intervention et la connaissent bien (CE).

Insertion de la sonde d'alimentation

- Les données probantes sont insuffisantes pour recommander la GEP ou la GRG comme intervention à privilégier pour la gastrostomie (niveau C). On dispose de données probantes de faible qualité selon lesquelles la GRG serait plus sécuritaire chez les patients qui ont une insuffisance respiratoire étant donné qu'elle ne requiert pas de sédation substantielle (CE).

- Une fois la sonde d'alimentation en place, un médecin d'expérience (endoscopiste ou radiologiste) devrait être rapidement disponible en cas de complications immédiates ou tardives liées à la sonde. Une diététiste devrait être disponible pour un soutien régulier concernant la nutrition entérale prescrite (CE).

- La sonde nasogastrique (SNG) n'est pas l'option à privilégier à long terme et devrait être réservée aux patients chez qui aucune autre approche n'est envisageable quand la nutrition entérale demeure souhaitable (niveau C).

Modifications diététiques et soutien nutritionnel

- On peut utiliser des régimes hypercaloriques pour améliorer les indicateurs nutritionnels (niveau B) et possiblement la survie (niveau C). Les régimes hypercaloriques-hyperglucidiques pourraient être préférables aux régimes hypercaloriques-hyperlipidiques (niveau B).

- L'alimentation parentérale est une source nutritionnelle envisageable chez les patients à qui on n'arrive pas à poser une sonde entérale; son utilisation doit être réservée à des cas exceptionnels (EC).

\section{Thromboembolie veineuse}

- Le risque de TEV est probablement plus élevé chez les patients atteints de SLA et ce risque semble accru lorsque l'atteinte a débuté aux membres inférieurs, chez des patients dont la mobilité est réduite (CE).

- Il faut encourager les professionnels de la santé à envisager la TEV comme cause potentielle d'une douleur ou d'un œdème aux jambes d'apparition récente chez les patients atteints de SLA (CE).

- Il n'y a pas de données probantes à l'appui d'un dépistage de la thromboembolie asymptomatique chez les patients atteints de SLA (CE).

- La thromboprophylaxie n'a pas été évaluée chez les patients atteints de SLA et n'est pas recommandée chez ceux qui n'ont pas été hospitalisés (CE).

- En cas de TEV chez un patient atteint de SLA, il faut administrer des anticoagulants, conformément aux normes thérapeutiques en vigueur (CE).

\section{Gestion de la pharmacothérapie}

- Les médecins de premier recours et les spécialistes devraient procéder périodiquement à une revue des médicaments et envisager l'arrêt de ceux qui ne sont pas indispensables (CE).

- Il faut maintenir les médicaments qui servent à traiter les symptômes (CE).

- Les médicaments de prévention primaire devraient être cessés si leur durée d'effet est plus longue que la survie prévisible (CE).

- On peut rassurer les patients et les professionnels de la santé sur le fait que les statines ne semblent pas contribuer au développement de la SLA (niveau B).

- Les données sont insuffisantes pour recommander l'arrêt des statines chez tous les patients atteints de SLA. L'arrêt des statines pourrait être envisagé selon la survie prévisible des patients et leur risque cardiovasculaire (CE).

\section{Prise en charge des symptômes}

Douleur

- On sait que la douleur est fréquente chez les patients atteints de la SLA et qu'elle a plusieurs étiologies possibles (CE).

- Les patients doivent être interrogés régulièrement au sujet de leurs symptômes douloureux. La douleur doit être régulièrement évaluée et les traitements adaptés à la cause spécifique (CE). 
Tableau 1 (partie 4 de 6): Recommandations assorties du niveau de données probantes pour la prise en charge des patients atteints de sclérose latérale amyotrophique*

\section{Recommandations}

\section{Prise en charge des symptômes (continu)}

Fasciculations

- Chez la plupart des patients, les fasciculations n'ont pas besoin d'une prise en charge médicamenteuse (CE).

- Si les fasciculations provoquent une détresse substantielle, on peut envisager l'utilisation de la gabapentine (niveau C).

Sialorrhée

- Les anticholinergiques sont le traitement de première intention de la sialorrhée. Le choix du médicament doit être adapté aux caractéristiques du patient (CE).

- Si un anticholinergique se révèle inefficace, on peut en essayer un autre (CE).

- On peut utiliser la succion buccale comme traitement d'appoint pour la sialorrhée (CE).

- La toxine botulinique est un traitement efficace de la sialorrhée chez les patients atteints de la SLA (niveau A). On peut l'utiliser comme traitement de deuxième intention et l'envisager après l'insertion d'une sonde d'alimentation en raison d'un risque théorique d'aggravation des problèmes de déglutition et d'intégrité des voies respiratoires (CE).

- L'irradiation locale des glandes salivaires est une option pour venir à bout de la sialorrhée comme traitement de deuxième ou de troisième intention (niveau C).

Syndrome pseudobulbaire

- Il faut informer les patients et les familles que le syndrome pseudobulbaire est un symptôme de la SLA et pas nécessairement un symptôme de dépression ou d'atteinte cognitive (CE).

- Le syndrome pseudobulbaire ne requiert aucun traitement à moins que le patient ressente de la détresse (CE).

- Si un traitement est justifié, des médicaments qui soulagent certains symptômes concomitants (p. ex., on peut envisager l'amitriptyline pour les troubles du sommeil et de l'humeur et les ISRS pour la dépression) peuvent être envisagés (CE).

- Le dextrométhorphane (20 mg) allié à de la quinidine (10 mg) peut servir au traitement du syndrome pseudobulbaire (niveau B).

Spasticité

- Souvent, les étirements peuvent être utiles pour la gestion de la spasticité (niveau C).

- Si une prise en charge pharmacologique de la spasticité s’impose, le baclofène, la tizanidine, la toxine botulinique, les benzodiazépines et les cannabinoïdes pourraient être envisagés (CE).

- Les données probantes sont insuffisantes pour recommander le baclofène intrathécal pour le traitement de la spasticité chez les patients atteints de la SLA (CE).

Crampes

- Il faut distinguer les crampes musculaires des autres causes de douleur (CE).

- La prise en charge de première intention devrait reposer notamment sur le soda tonique, la gabapentine et le baclofène (CE).

- Le traitement de deuxième intention pourrait inclure la quinine, le lévétiracétam et la mexilétine (CE).

Dépression

- La dépression qui accompagne la SLA doit être traitée, car elle affecte substantiellement le bien-être du patient (CE).

- Les ISRS ou les IRSN peuvent être utilisés pour traiter la dépression chez les patients atteints de la SLA (CE).

- Un soutien non pharmacologique peut être envisagé, p. ex., avec l'aide de psychologues, travailleurs sociaux, psychiatres ou accompagnants spirituels (CE).

Anxiété

- L'anxiété qui accompagne la SLA doit être traitée, car elle affecte substantiellement le bien-être des patients (CE).

- Il est important de déterminer si l'anxiété est due à l'insuffisance respiratoire et, le cas échéant, traiter cette dernière en conséquence (CE).

- En présence de dépression concomitante, la prescription d'un IRSR s'impose (CE).

- Les benzodiazépines peuvent exacerber l'insuffisance respiratoire (CE).

- Un soutien non pharmacologique peut être envisagé, p. ex., avec l'aide de psychologues, travailleurs sociaux, psychiatres ou accompagnants spirituels (CE).

Insomnie

- L'insomnie peut avoir plusieurs causes, telles que l’insuffisance respiratoire et la dépression, qui devraient être évaluées de manière appropriée (CE).

- On peut envisager des épreuves respiratoires et des études du sommeil pour déterminer le type et la cause de l'insomnie (CE).

- La prise en charge pharmacologique de l'insomnie dépendra de sa cause (CE).

Fatigue

- Des causes réversibles de la fatigue méritent d'être envisagées, telles qu'insuffisance respiratoire, troubles du sommeil, dépression, effets indésirables des médicaments et utilisation du riluzole (CE).

- Chez les patients sous riluzole qui présentent de la fatigue, on peut envisager d'en réduire la dose ou de le cesser (niveau C).

- On peut envisager de demander à un ergothérapeute d'enseigner aux patients des techniques de conservation de l'énergie (CE). 


\section{Recommandations}

\section{Dysarthrie}

- Les patients dysarthriques devraient être suivis régulièrement en orthophonie pour qu'on intervienne sans délai en cas de problème de communication (CE).

- Des appareils de communication, amplificateurs ou autres, devraient être offerts aux patients admissibles dès les premiers stades de la maladie (CE). Les patients aux stades plus avancés bénéficieront aussi de dispositifs et de stratégies pour aider à la communication (CE).

- Le choix du dispositif de communication doit être adapté aux besoins et aux capacités du patient (CE). Les patients qui ont une atteinte cognitive pourraient avoir besoin de stratégies individualisées (CE).

- Les stratégies de communication (amplificateurs et autres) pourraient réduire le stress des proches aidants (CE).

- L'amplification vocale devrait être offerte aux patients qui ont des problèmes de projection vocale (CE).

- Des banques vocales devraient être offertes à certains patients (CE).

- Offrir un accès à différents modes de communication, notamment les médias sociaux, peut être propice à l'autonomie, à la participation sociale et à une meilleure QdV (CE).

\section{Exercices}

- Aux premiers stades de la SLA, un programme d'exercices réguliers d'intensité modérée est probablement bénéfique pour le fonctionnement et la QdV (niveau B). Un programme d'exercices personnalisés (musculation et entraînement aérobique) devrait être suggéré aux patients qui y sont aptes (CE) :

- Il faut encourager un effort sous-maximal en termes de résistance.

- Les activités physiques d'intensité modérée sont celles qui entraînent une légère transpiration ou une accélération de la fréquence respiratoire.

- L'exercice d'intensité modérée est bien toléré et n'est pas nuisible chez les patients atteints de la SLA (niveau B):

- La fatigue ou la douleur après l'exercice devrait rentrer dans l'ordre en 30 minutes et ne pas interférer avec les activités quotidiennes; autrement, le programme d'exercices devrait être ajusté.

- Un programme d'étirements et d'amplitude de mouvement est recommandé dans les cas de spasticité (niveau C) et de douleur (CE) et pour prévenir les contractures (CE).

- Les exercices d'étirement et d'amplitude de mouvement peuvent être faits de manière autonome (mouvement actif), avec de l'aide (mouvement passif) ou en association (actif et passif).

\section{Fonctions cognitives et comportementales}

- Le dépistage des atteintes cognitive et comportementale doit être effectué chez les patients atteints de SLA au début de la maladie (niveau B).

- Si à un moment ou à un autre, on s'inquiète d'un problème cognitif ou comportemental, des évaluations spécifiques doivent être effectuées chez la personne, les membres de sa famille et ses proches aidants, selon le cas (CE).

- On ne dispose d'aucune étude sur l'utilisation d'agents pharmacologiques pour la prise en charge des troubles cognitifs ou comportementaux chez les patients atteints de la SLA.

- Étant donné que la présence d'une démence frontotemporale nuit à la survie, la planification préalable des soins est indiquée au début de la maladie (CE).

- La présence d'atteinte cognitive ou comportementale ne devrait pas nécessairement empêcher la recommandation d'une VNE ou d'une gastrostomie. Toutefois, les défis de l'observance thérapeutique dans les cas d'atteinte cognitive ou comportementale doivent faire l'objet de discussions avec le patient et ses proches avant d'appliquer l'intervention (CE).

- Une approche multidisciplinaire peut être envisagée pour la prise en charge des comportements particulièrement problématiques. La participation de spécialistes du comportement (ergothérapeute ou psychologue) ou de psychiatres peut être envisagée (CE).

\section{Proches aidants}

- Les professionnels de la santé doivent être attentifs aux besoins et au bien-être émotionnel des proches aidants et ces derniers doivent être impliqués dans la planification des soins en tenant compte de l'incidence prévisible de la SLA sur le patient et ses proches (CE).

- Les cliniques multidisciplinaires devraient être au courant du stress financier que subissent les proches aidants et les renseigner sur les programmes d'aide existants, le cas échéant (CE).

- Évaluer le fardeau des proches aidants, leurs stratégies d'adaptation, leur état d'esprit et les dynamiques familiales permet d'identifier ceux qui auront besoin de répit et de services de soutien. Les sociétés locales de SLA mettent parfois des ressources à la disposition des proches et des proches aidants (EC).

\section{Soins palliatifs}

Planification

- Le personnel clinique, des professionnels des soins palliatifs et les médecins de famille peuvent offrir les soins palliatifs aux patients atteints de SLA tout au long de la maladie (CE).

- Les soins palliatifs doivent être offerts en cas de détresse physique (douleur, dysphagie ou dyspnée), psychosociale ou existentielle grave (CE).

- Pour assurer la continuité des soins intégrés, des services communautaires de soins palliatifs peuvent être débutés avant les stades avancés de la SLA (EC). 
Tableau 1 (partie 6 de 6): Recommandations assorties du niveau de données probantes pour la prise en charge des patients atteints de sclérose latérale amyotrophique*

\section{Recommandations}

\section{Soins palliatifs (continu)}

Traitements

- Face à un patient atteint d'essoufflement, le médecin doit clarifier si l'objectif des soins est de prolonger la vie ou d'améliorer le confort, et assurer une mort en douceur (CE).

- Les médecins doivent évaluer l'essoufflement et atténuer les facteurs en cause, tels que les sécrétions buccales et l'anxiété (CE).

- Des opioïdes peuvent être administrés pour soulager l'essoufflement (CE).

- Un flux d'air au visage peut aider à soulager l'essoufflement (CE).

- Les conversations sur la planification préalable des soins doivent être amorcées au début de la maladie et chaque fois que le patient pose des questions. Une discussion sur la planification préalable des soins et les objectifs des soins devrait faire partie du suivi régulier de la SLA (CE).

- Les patients doivent être encouragés à discuter de leurs volontés au sujet de leurs soins de fin de vie avec les membres de leur famille et leurs proches aidants (CE).

- Les soins palliatifs devraient être intégrés à la prise en charge des patients dès avant la phase terminale de la SLA (CE).

- L'utilisation de la VNE et des sondes de gastrostomie doit être maintenue avec les soins palliatifs pour le soulagement des symptômes et pour la QdV, selon les préférences du patient (EC).

Arrêt du soutien respiratoire

- L'arrêt du soutien respiratoire continu ne doit avoir lieu qu'après consultation et planification avec un professionnel de la santé ayant des connaissances dans ce domaine, ainsi qu'en matière de sédation palliative (CE).

- Il faut avoir obtenu une maîtrise adéquate des symptômes prévisibles au moyen d'opioïdes et de benzodiazépine avant d'arrêter la ventilation (CE).

- Il faut offrir à la famille et aux professionnels de la santé un soutien psychosocial et des rencontres de suivi (CE).

Deuil

- Un soutien psychosocial pour les proches aidants endeuillés doit être offert. La discussion sur le deuil et un soutien adéquat à cet égard peuvent débuter avant même le décès du patient (CE).

Aide médicale à mourir (AMM)

- Des discussions au sujet de l'AMM doivent être menées par un médecin ou une infirmière praticienne, conformément aux lignes directrices régionales (CE).

- Les médecins qui soignent des patients atteints de SLA doivent fournir l'accès à des renseignements sur l'AMM lorsqu'elle est demandée (CE).

- On ne devraient pas présumer que les questions sur l'AMM équivalent à une demande d'AMM. Toutefois, les questions à ce sujet se prêtent aussi à un dialogue sur les soins de fin de vie et la planification préalable des soins (CE).

- Les patients qui demandent une AMM devraient bénéficier de soins palliatifs et de soutien (EC).

Don d'organes

- Les patients atteints de la SLA peuvent être acceptés comme donneurs d'organe pleins, moyennant approbation de l'organisme local responsable (CE).

- Les patients atteints de la SLA ne peuvent pas faire de dons de tissus tels que la cornée, la peau ou les os (CE).

- Les cliniques devraient orienter les demandes relatives à de tels dons vers l'organisme provincial responsable (CE).

- À leur décès, les patients peuvent donner leurs tissus pour la recherche sur la SLA (EC).

Remarque : ALSFRS-R = Amyotrophic Lateral Sclerosis Functional Rating Scale - Revised, $\mathrm{AMM}=$ aide médicale à mourir, $C E=$ consensus des experts, CVF = capacité vitale forcée, DÉQT = dépense énergétique quotidienne totale, DPT = débit de pointe à la toux, ÉEFO-D = évaluation endoscopique par fibre optique de la déglutition, GEP = gastrostomie endoscopique percutanée, $\mathrm{GRG}=$ gastrostomie radioguidée, IEM = insufflation-exsufflation mécanique, IMC = indice de masse corporelle, ISRS = inhibiteur sélectif du recaptage de la sérotonine, $\mathrm{pCO} \mathrm{C}_{2}=$ pression partielle du gaz carbonique, $\mathrm{PIM}=$ pression inspiratoire maximale, $\mathrm{PIN}=$ pression inspiratoire nasale, $\mathrm{PPS}=$ planification préalable des soins, $\mathrm{QdV}=$ qualité de vie, $\mathrm{SLA}=\mathrm{sclérose}$ latérale amyotrophique, TEV = thromboembolie veineuse, $\mathrm{VNE}=$ ventilation non effractive.

*Voir encadré $n^{\circ} 2$ pour les critères des niveaux de preuve à l'appui des recommandations.

†Si les épreuves pulmonaires ne sont pas fiables (c.-à-d., chez des patients qui ont un syndrome bulbaire ou une grave atteinte cognitive), les médecins doivent uniquement se fier aux symptômes ou à d'autres mesures de dépistage de l'insuffisance respiratoire.

łLe domicile du patient est son lieu de résidence (maison, appartement ou établissement de soins de longue durée).

\section{Multidisciplinarité des soins}

Les patients atteints de SLA devraient être suivis régulièrement dans une clinique multidisciplinaire de SLA de concert avec leurs médecins traitants. Les soins multidisciplinaires devraient être prodigués par une équipe composée de médecins et d'autres professionnels de la santé chargés de veiller à divers paramètres, notamment communication, nutrition, déglutition, mobilité, activités de la vie quotidienne, soins respiratoires, capacité cognitive, enjeux psychosociaux, traitements médicaux et soins de fin de vie. Chez les patients suivis dans une clinique multidisciplinaire on a enregistré de meilleurs résultats, y compris amélioration de la survie, réduction des hospitalisations, utilisation accrue d'équipements adaptés et meilleure qualité de vie, comparativement aux patients qui n'étaient pas suivis dans ce type de clinique ${ }^{31-33}$. Selon une étude de cohorte prospective, les patients suivis dans une clinique multidisciplinaire vivaient 7,5 mois de plus que les patients suivis dans une clinique de neurologie générale ${ }^{32}$.

La télémédecine et la surveillance à distance sont faisables et pourraient compléter les soins multidisciplinaires en clinique ${ }^{34}$. La prise en charge des patients atteints de SLA devrait être une 
collaboration entre le médecin de famille et la clinique de SLA, le personnel de cette dernière se montrant disponible pour des consultations à distance entre les visites des patients.

\section{Soins respiratoires}

Le groupe responsable des lignes directrices émises par la Société canadienne de thoracologie (SCT) a récemment révisé la prise en charge respiratoire des patients atteints de SLA sous ventilation mécanique à domicile ${ }^{35}$. Nous avons décidé de faire concorder nos recommandations sur la prise en charge respiratoire (tableau 1, figure 1) avec celles de la SCT. Nous y avons cependant fait quelques ajouts, notamment un énoncé sur l'évitement de l'oxygénothérapie pour les symptômes respiratoires chez les patients atteints de SLA, le moment de l'instauration des interventions et la gestion des sécrétions. Nous avons aussi jugé important d'ajuster le critère de capacité vitale forcée (CVF) minimale pour l'instauration de la ventilation

\section{A) Ventilation}

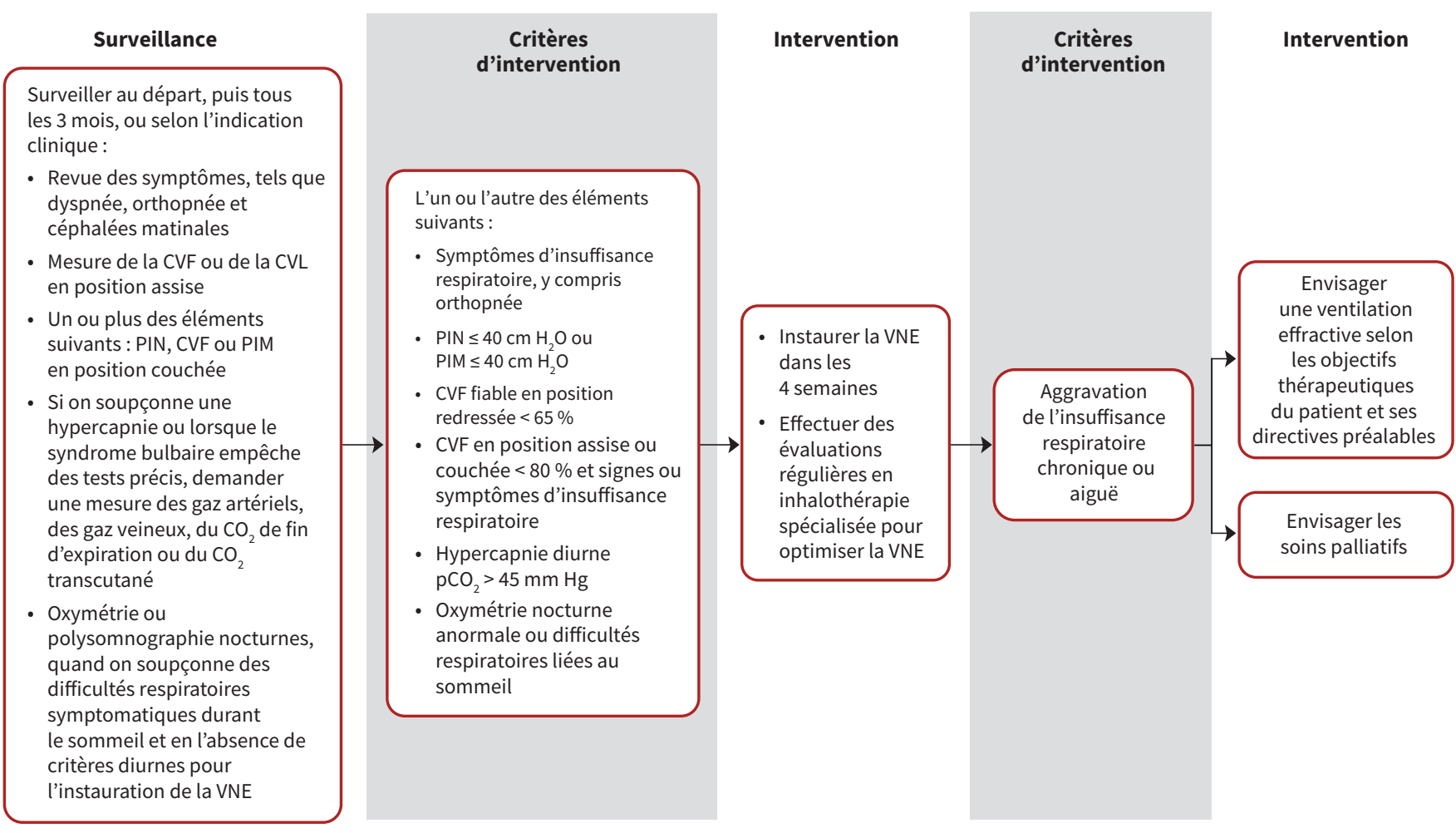

B) Clairance des voies respiratoires

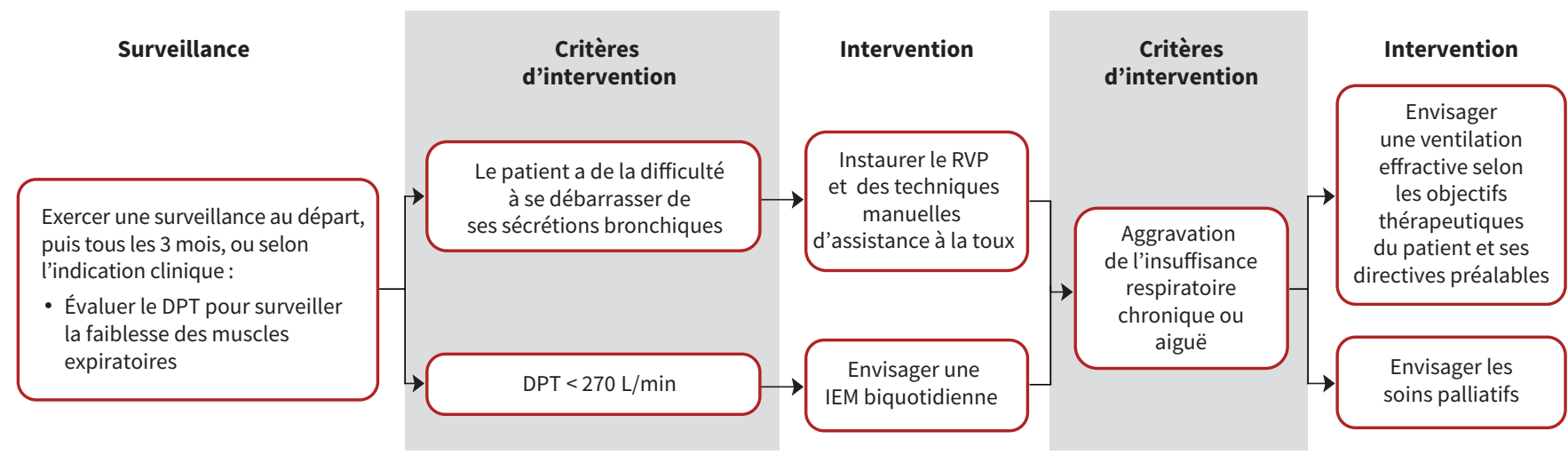

Figure 1 : Arbre décisionnel respiratoire : Sommaire des recommandations pour les soins respiratoires chez les patients atteints de sclérose latérale amyotrophique (SLA), y compris ventilation (A) et clairance des voies respiratoires (B). Remarque : CVF = capacité vitale forcée, $C V L=$ capacité vitale lente, $\mathrm{DPT}=$ débit de pointe à la toux, $\mathrm{H}_{2} \mathrm{O}=$ eau, IEM = insufflation-exsufflation mécanique, $\mathrm{pCO}_{2}=$ pression partielle du gaz carbonique, $\mathrm{PIM}=$ pression respiratoire maximale, $\mathrm{PIN}=$ pression inspiratoire nasale, $\mathrm{RVP}=$ recrutement de volume pulmonaire, $\mathrm{VNE}=$ ventilation non effractive. 
non effractive chez les patients asymptomatiques à $65 \%$ des valeurs prévues, par rapport à la recommandation de la SCT (50\%), en raison des données probantes existantes selon lesquelles l'instauration précoce améliore la survie ${ }^{36}$. Les patients qui ont une CVF supérieure à $65 \%$ de la valeur prévue peuvent débuter une ventilation non effractive si tout autre critère à cet effet est présent, conformément aux lignes directrices de la SCT. Notre groupe a aussi unanimement jugé que si les critères d'instauration de la ventilation non effractive sont présents, il faudrait la débuter dans les 4 semaines.

Il est important de reconnaître que la ventilation non effractive peut modifier la trajectoire naturelle de la maladie. Par exemple, une dépendance accrue à l'endroit de la ventilation non effractive transforme cette dernière en une intervention de maintien des fonctions vitales. En effet, chez les patients dépendants de la ventilation non effractive, cette dernière prévient la mort naturelle aussi longtemps qu'une décision n'est pas délibérément prise de cesser la ventilation. Les patients devraient être informés qu'ils pourraient devoir prendre une décision délibérée au sujet de l'arrêt éventuel de la ventilation, à moins de vouloir prolonger leur survie.

La gestion des sécrétions est souvent difficile chez les personnes atteintes de SLA, et c'est une cause de détresse, de réduction de la qualité de vie et de dysfonction respiratoire. Les lignes directrices de la SCT ne mentionnent pas explicitement la clairance des voies respiratoires. Pour aborder cet important enjeu, nous avons passé en revue les données probantes disponibles et l'expérience clinique. Nous recommandons des techniques de récupération du volume respiratoire dès que les patients ont des sécrétions encombrantes ou présentent des difficultés à éliminer les sécrétions. Ces techniques peuvent être combinées avec des techniques manuelles d'assistance à la toux et effectuées par les patients seuls ou avec l'aide de professionnels de la santé. Si les patients présentent un débit de pointe à la toux (DPT) anormal (<270 L/min), l'insufflation-exsufflation mécanique biquotidienne est à envisager pour éliminer les sécrétions, et plus fréquemment lors d'infections respiratoires aiguës.

Nous convenons aussi qu'il est essentiel de fournir un soutien respiratoire adéquat là où réside le patient avec la ventilation non effractive et l'insufflation-exsufflation mécanique à des fins d'enseignement, de titrage et de résolution de problèmes, indépendamment du lieu de résidence du patient (domicile, établissement de soins de longue durée ou centre de soins palliatifs).

\section{Nutrition}

Les recommandations nutritionnelles (tableau 1, figure 2) suivent passablement celles que l'on retrouve dans la ligne directrice de l'American Academy of Neurology (AAN) ${ }^{6}$. Les différences par rapport aux recommandations de l'AAN incluent l'ajout de l'énoncé

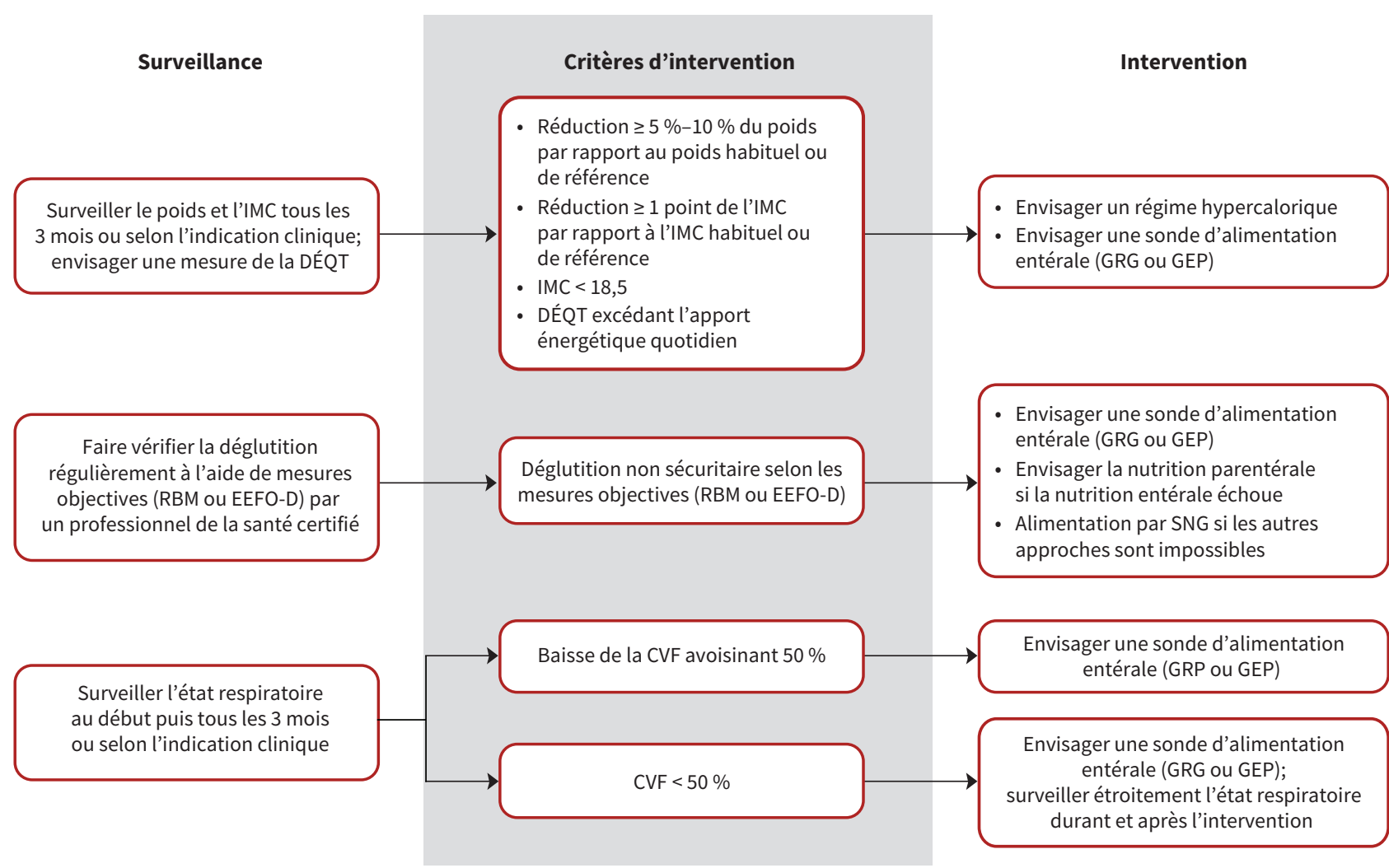

Figure 2 : Arbre décisionnel nutritionnel : Sommaire des recommandations pour la prise en charge nutritionnelle des patients atteints de sclérose latérale amyotrophique (SLA). Remarque : CVF = capacité vitale forcée, DÉQT = dépense énergétique quotidienne totale, EEFO-D = évaluation endoscopique par fibre optique de la déglutition, GEP = gastrostomie endoscopique percutanée, GRG = gastrostomie radioguidée, IMC = indice de masse corporelle, RBM = repas baryté modifié, SNG = sonde nasogastrique. 
du consensus des experts sur le délai maximum de 4 semaines envisageable avant la pose d'une sonde d'alimentation une fois les critères présents, et d'un énoncé concernant le suivi approprié après l'insertion pour prévenir les complications immédiates ou tardives. Les recommandations incluent aussi un énoncé sur la composition de l'alimentation et précisent que des régimes hypercaloriques peuvent être utilisés pour améliorer les indicateurs nutritionnels et possiblement la survie ${ }^{37,38}$. Les régimes hypercaloriques-hyperglucidiques pourraient être préférables aux régimes hypercaloriques-hyperlipidiques ${ }^{39}$.

\section{Thromboembolie veineuse}

Les patients atteints de SLA ${ }^{40,41}$ sont probablement exposés à un risque plus grand de thromboembolie veineuse (TEV). Le risque semble aggravé lorsque la SLA affecte d'abord les membres inférieurs et que la mobilité diminue ${ }^{40}$. Malgré ce risque accru, aucune étude n'appuie le recours à la thromboprophylaxie primaire. Pour l'instant, cette dernière n'est pas recommandée parce qu'on ignore le rapport risque-bénéfice des conséquences potentiellement négatives d'une chute par rapport à la prévention de la TEV chez les patients atteints de SLA.

\section{Gestion de la pharmacothérapie}

Lorsque les patients consultent dans une clinique de SLA, ils prennent souvent plusieurs médicaments qui ne sont peutêtre pas tous indispensables, particulièrement si l'on tient compte de la survie moyenne des patients atteints de SLA. Selon le consensus des experts, nous avons rédigé plusieurs énoncés sur l'importance de réviser régulièrement les médicaments du patient et de suggérer l'arrêt de tout agent non essentiel, qui ne soulage pas ses symptômes ou ne lui procure aucun bienfait thérapeutique approprié compte tenu de son espérance de vie.

\section{Soulagement des symptômes}

Les patients atteints de SLA ont souvent plusieurs types de symptômes incommodants qui nuisent gravement à leur qualité de vie, notamment douleur, fasciculations, sialorrhée, syndrome pseudobulbaire, spasticité, crampes, dépression, anxiété, insomnie et fatigue. Plusieurs essais cliniques ont exploré le traitement de la sialorrhée ${ }^{42}$ et du syndrome pseudobulbaire ${ }^{43}$. Toutefois, la prise en charge de la plupart des symptômes de la SLA n'a pas été rigoureusement évaluée. C'est pourquoi la majeure partie des recommandations pour la prise en charge des symptômes ont été établies par un consensus d'expert et s'appuient sur des suggestions formulées dans la littérature sur la SLA et les soins palliatifs. Le coût des traitements et leur accessibilité ont influencé le classement de nos recommandations et nous avons accordé plus de poids à ces 2 critères qu'aux données probantes directes si un traitement reposant sur des données probantes était coûteux. Nos recommandations n'ont pas inclus le cannabis pour le traitement de symptômes spécifiques de la SLA, en raison de l'absence de données probantes dans la littérature. Mais le groupe de travail sait que le cannabis est utilisé pour gérer plusieurs des symptômes de la SLA.

\section{Dysarthrie}

La capacité d'exprimer ses idées et ses besoins est vitale pour tous. La SLA nuit souvent à la capacité de communiquer verbalement en raison de la dysarthrie ${ }^{2}$. Il existe des outils non technologiques pour faciliter la communication, dont les tableaux de lettres ou de pictogrammes, et des outils technologiques, tels que les synthétiseurs vocaux et les systèmes à commande oculaire. À mesure que les pertes fonctionnelles se manifestent chez la personne atteinte de SLA, certains modes de communication pourraient ne plus être adaptés. Fournir l'accès à différents modes de communication, y compris les médias sociaux, peut préserver l'autonomie et la participation à la vie sociale, et améliorer la qualité de vie. Les dispositifs d'aide à la communication ${ }^{44}$ peuvent aussi être utiles aux proches aidants; pour ces derniers, le fardeau a été allégé par le recours à des systèmes de commande oculaire ${ }^{45}$.

\section{Exercices}

La recherche sur l'exercice pour les patients atteints de la SLA n'a pas révélé qu'il était nuisible et selon certaines données probantes, il conférerait un avantage potentiel aux patients aux plans de leur capacité fonctionnelle et de leur qualité de vie ${ }^{46,47}$. Un programme personnalisé d'exercices, incluant musculation et entraînement aérobique, devrait être préconisé pour les patients qui y sont aptes. Un programme régulier d'étirements et d'amplitude de mouvement est recommandé pour lutter contre la spasticité et la douleur, et prévenir les contractures.

\section{Fonctions cognitives et comportementales}

Une dysfonction frontotemporale s'observe chez environ $50 \%$ des patients atteints de SLA ${ }^{3}$. La dysfonction frontotemporale peut s'accompagner d'une atteinte cognitive ou comportementale qui, chez $20 \%$ des patients, est suffisamment grave pour porter le nom de démence ${ }^{3}$. Même s'il existe plusieurs outils pour faire le dépistage d'une atteinte cognitive ou comportementale, aucun n'est standardisé. Pour l'instant, il n'existe aucun traitement médicamenteux efficace pour l'atteinte cognitive ou comportementale associée à la SLA. Une approche multidisciplinaire est envisageable pour la prise en charge des comportements particulièrement problématiques.

La présence d'une dysfonction exécutive ou de démence chez les patients atteints de la SLA est associée à une piètre survie ${ }^{48}$. L'atteinte cognitive ou comportementale ne constitue pas nécessairement une contre-indication à la ventilation non effractive ou à la pose d'une sonde de gastrostomie. Mais avant d'aller de l'avant avec ces interventions chez un patient souffrant d'une atteinte cognitive ou comportementale, il faut toutefois aborder les éventuels problèmes d'observance thérapeutique avec le patient lui-même et ses proches.

\section{Proches aidants}

Les soins à la personne atteinte de SLA représentent un lourd fardeau pour les proches aidants. De nombreuses études ont démontré l'incidence de la SLA sur la qualité de vie des proches aidants et les multiples facettes de ce rôle ${ }^{49}$, mais également la valeur inestimable de la prestation des soins. La progression de l'invalidité ${ }^{50}$ (score ALSFRS-R) et de l'atteinte cognitive alourdit le 
fardeau pour les proches aidants ${ }^{51}$. Les interventions visant à atténuer cette incidence ont été trop peu étudiées pour qu'on puisse formuler des recommandations spécifiques. Les personnes atteintes de SLA sont conscientes du fardeau imposé à leurs proches aidants et elles en souffrent aussi ${ }^{52}$. Les professionnels de la santé doivent donc être attentifs au bien-être physique et émotionnel des proches aidants, et les faire participer à la planification en vue de l'incidence de la SLA sur le patient et sur eux-mêmes.

\section{Soins palliatifs}

Les experts sont en faveur de l'intégration précoce des soins palliatifs pour les patients atteints de SLA ${ }^{8,53}$. Mais la question des soins palliatifs et des soins de fin de vie est délicate à aborder et n'est pas reçue de la même façon par tous les patients ${ }^{54}$. Il faut donc tenir compte de l'évolution des besoins et des attentes des patients pour pouvoir amener hâtivement la question des soins palliatifs ${ }^{55}$. Les experts conseillent à tout le moins d'engager la discussion sur les soins palliatifs si le sujet est amené par les patients ou les proches aidants et s'ils sont indiqués en raison de l'aggravation de la maladie ou de l'invalidité53.

La planification préalable des soins permet au patient de faire connaître ses volontés en la matière avant d'être trop malade et incapable de communiquer. Selon certaines données probantes, il est préférable d'amorcer ces discussions lorsque le patient a accepté que la mort est inéluctable ${ }^{56}$. Mais les médecins sont souvent réticents à en parler de crainte que ce soit perçu comme une indication que la mort est imminente ${ }^{57}$. Les outils standardisés pour la planification préalable des soins sont utiles pour mener ces discussions, et non pour donner des directives spécifiques ${ }^{56,58,59}$. Ces outils peuvent être intégrés dans le suivi de routine de la SLA pour ouvrir la porte à la discussion, en tenant compte de l'état de préparation du patient et de sa façon de prendre des décisions.

Le 17 juin 2016, l'aide médicale à mourir a été légalisée au Canada. Nous avons formulé des recommandations spécifiques sur la façon de répondre aux demandes d'AMM, tant pour aider le patient dans son choix de fin de vie que pour guider les médecins dans cette nouvelle pratique, qui peut soulever une incertitude clinique et de l'inconfort chez certains.

Nous formulons aussi des recommandations sur la possibilité d'effectuer des dons d'organe lors du décès et de la marche à suivre le cas échéant.

\section{Méthodes}

Le concept qui sous-tend cette ligne directrice a été développé par la Société canadienne de la SLA avec l'aide de médecins canadiens qui soignent des patients atteints de cette maladie au sein du Réseau canadien de recherche sur la SLA, le Canadian ALS Research Network (CALS), désormais fusionné avec la Société canadienne de la SLA. La ligne directrice a été rédigée à l'aide de l'outil Guideline International Network-McMaster Guideline Development Checklist ${ }^{60}$, qui explique toutes les étapes de la préparation d'une ligne directrice, notamment la planification, le libellé des recommandations, leur mise en œuvre et leur évaluation. Une description complète de la méthodologie qui a été utilisée est accessible dans la ligne directrice intégrale (www.als.ca/bpr-appendix).

\section{Composition du comité de rédaction des lignes directrices}

Un groupe de travail formé de 13 médecins canadiens qui soignent des personnes atteintes de la SLA (les auteurs), présidé par C.S., a dirigé la préparation de cette ligne directrice. Des neurologues et des physiatres actifs au sein du Réseau canadien de recherche sur la SLA qui pouvaient représenter la diversité géographique du Canada ont été invités à participer au groupe de travail. Des médecins qui avaient une expérience dans la rédaction de lignes directrices ont été particulièrement encouragés à participer. Le groupe de travail incluait aussi un gastroentérologue (D.L.) et une pneumologue (A.T.) connaissant bien la SLA. Au début du processus de préparation de la ligne directrice, 2 autres médecins canadiens qui soignent des personnes atteintes de la SLA ont été impliqués, mais ils se sont retirés du projet faute de temps.

\section{Choix des questions clés}

En 2014, pour sélectionner les questions cliniques d'intérêt en vue de la rédaction de la ligne directrice, nous avons interrogé les médecins et le personnel des 19 cliniques multidisciplinaires canadiennes de SLA au moyen d'un sondage envoyé par courriel. Le sondage incluait une liste des questions clés utilisées par l'American Academy of Neurology (AAN) et l'European Network for the Cure of ALS lors du choix de leurs paramètres de pratique ${ }^{6,7,8}$, ainsi que d'autres questions tirées de la propre expérience clinique des membres du groupe de travail. Nous avons demandé aux répondants d'évaluer la pertinence de ces questions en vue de leur inclusion dans la ligne directrice.

Les questions utilisées pour la revue de la littérature sont celles que les participants ont jugées importantes lors du sondage; le groupe de travail les a précisées davantage. Les enjeux cliniques sélectionnés ont été regroupés par thèmes, notamment : annonce du diagnostic, traitement modificateur de la maladie, soins multidisciplinaires, soins respiratoires, nutrition, traitement des symptômes, atteinte cognitive, risque de thromboembolie veineuse (TEV), exercices, soins palliatifs et soutien aux proches aidants.

\section{Recherche documentaire}

En 2015, le Centre for Effective Practice, une firme de consultants experts en rédaction de lignes directrices, a procédé à des interrogations de la littérature sur les questions cliniques sélectionnées dans les bases de données MEDLINE, Embase et CINAHL. Le centre a dégagé les mots-clés pour la recherche sur chacune des questions cliniques en faisant un survol de ceux qui avaient été utilisés pour la ligne directrice de l'AAN ${ }^{6,7}$ et en consultation avec le groupe de travail. Le centre a procédé à une seconde interrogation de la littérature en décembre 2018 pour recenser des articles publiés après la recherche initiale de 2015. Pour les questions cliniques abordées dans la ligne directrice de l'AAN, les interrogations de la littérature se sont limitées aux publications datant de 2007 à décembre 2018. Pour les nouvelles questions cliniques qui n'avaient pas été abordées dans les lignes directrices de l'AAN et de l'European Network for the Cure of ALS, les interrogations de la littérature se sont limitées aux publications de 1998 à décembre 2018. Les stratégies d'interrogation sont décrites à l'annexe $A$ de la ligne directrice intégrale (www.als.ca/bpr-appendix). 
Encadré $n^{\circ} 1$ : Critères d'évaluation des études thérapeutiques ${ }^{\star 61}$

\begin{tabular}{|c|c|}
\hline Classe & Description \\
\hline I & $\begin{array}{l}\text { - Essai clinique randomisé et contrôlé (ERC) auprès d'une population représentative } \\
\text { - Évaluation des paramètres masquée ou objective } \\
\text { un cajustement statistique approprié pour tenir compte des différences } \\
\text { - Également requis: } \\
\text { a. Assignation à l'insu } \\
\text { b. Paramètres principaux clairement définis } \\
\text { c. Critères d'exclusion et d'inclusion clairement définis } \\
\text { d. Prise en compte adéquate des abandons (au moins } 80 \% \text { des participants inscrits ayant mené l'étude à terme) et permutations } \\
\text { avec des nombres suffisamment bas pour que le risque de biais soit minimal }\end{array}$ \\
\hline ॥ & $\begin{array}{l}\text { - Étude de cohorte présentant les critères a) à d) (voir classe I) ou ERC dépourvu de } 1 \text { ou } 2 \text { critères b) à d) (voir classe I) } \\
\text { - Toutes les caractéristiques de référence pertinentes sont présentées et substantiellement équivalentes entre les groupes traités, ou } \\
\text { - Évaluation des paramètres masquée ou objective }\end{array}$ \\
\hline III & $\begin{array}{l}\text { - Études contrôlées (y compris témoin historique naturel bien défini ou patients agissant comme leurs propres témoins) } \\
\text { - Description des différences de confusion majeure entre les groupes traités qui pourraient affecter les résultats } \\
\text { - Évaluation des résultats masquée, objective ou effectuée par quelqu'un qui n'est pas membre de l'équipe soignante }\end{array}$ \\
\hline IV & $\begin{array}{l}\text { - N'incluait pas de patients atteints de la maladie } \\
\text { - N'incluait pas de patients recevant différentes interventions } \\
\text { - Interventions ou paramètres non définis ou non acceptés } \\
\text { - Aucune mesure de l'efficacité ni précision statistique présentées ou calculables }\end{array}$ \\
\hline
\end{tabular}

*Modifié avec l’autorisation de l'AAN (American Academy of Neurology). 2011. Clinical Practice Guideline Process Manual, Éd. 2011 St. Paul, MN: The American Academy of Neurology. Énoncé de position de l'American Academy of Neurology. Accessible ici : www.aan.com/siteassets/home-page/policy-and-guidelines/guidelines/about-guidelines/11guidelinedevmanual_v408_web.pdf (consulté le 11 septembre 2020).

\section{Évaluation de la qualité des données}

Le groupe de travail a été scindé par thèmes en plusieurs équipes de 2 membres. Pour les questions relevant d'un thème majeur, 2 membres de chaque groupe ont passé en revue séparément les résumés recensés en fonction des critères d'inclusion et de leur pertinence visà-vis de la question clinique. Les critères d'inclusion étaient notamment : lignes directrices publiées sur la SLA et sur la ventilation, essais randomisés et contrôlés (ERC), études cas-témoins, études de cohorte, revues systématiques et méta-analyses. Les publications devaient être en langue anglaise ou française et disponibles en version intégrale. Les rapports de cas, articles de synthèse et publications disponibles uniquement sous forme de résumés ou d'actes de conférence et les thèses non publiées ailleurs ont été exclues. Les publications qui répondaient aux critères d'inclusion selon au moins un des réviseurs ont été entièrement passées en revue par le comité pour une analyse des critères d'inclusion et de la qualité des données et ont reçu une évaluation selon les critères modifiés de l'AAN Clinical Practice Guideline Process Manual (édition 2011) pour classer les études thérapeutiques (encadré $n^{\circ} 1$ ). Les tableaux sur les données probantes sont accessibles à l'annexe $\mathrm{B}$ de la ligne directrice intégrale (www.als.ca/bpr-appendix).

\section{Élaboration des recommandations}

Le groupe de travail s'est réuni régulièrement en personne au moins une fois l'an à Toronto, et lors de téléconférences périodiques pour discuter en détail des énoncés de la ligne directrice. Les comités ont tous rédigé une ébauche de leurs énoncés pour chaque question cli-
Encadré $n^{\circ} 2$ : Critères pour les niveaux de données probantes à l'appui des recommandations de la ligne directrice*

Niveau Type de données probantes

A

Au moins 2 études de classe I concordantes

B Au moins 1 étude de classe I ou 2 études de classe II concordantes

C Au moins 1 étude de classe II ou 2 études de classe III concordantes

Consensus Consensus entre les experts cliniques canadiens de la des experts sclérose latérale amyotrophique en l'absence de données (CE) probantes répondant aux critères des niveaux $A$ à $C$

*Voir encadré nº 1 pour la définition des classes d'études.

nique après avoir tenu compte du libellé des lignes directrices antérieures et des données probantes mises à jour ${ }^{6-8}$.

Le groupe de travail a passé en revue l'ébauche des énoncés et les a révisés selon une approche itérative, idéalement jusqu'à l'atteinte d'un consensus. En l'absence de consensus au sein du groupe de travail, nous aurions fondé les décisions sur un vote à la majorité des deux tiers (66\%). Toutefois, un consensus a été obtenu pour tous les énoncés, de sorte qu'un tel vote n'a pas eu lieu.

Nous avons attribué à chaque énoncé un niveau de preuve qui incluait le consensus des experts (encadré $n^{\circ} 2$ ). Le groupe de travail s'est exprimé nettement en faveur d'inclure des recommandations de pratiques optimales basées sur le consensus des experts 
en l'absence de données publiées, plutôt que de s'abstenir de toute recommandation. Étant donné le manque de données probantes tirées d'essais cliniques pour orienter les soins dans la SLA, les membres du groupe de travail ont jugé important d'offrir des recommandations pour guider la pratique, plutôt qu'une simple revue des données probantes. Nous avons formulé des énoncés consensuels d'experts basés sur la littérature concernant la SLA ne provenant pas d'essais cliniques, sur les données probantes concernant d'autres maladies ou sur la pratique clinique canadienne actuelle en matière de SLA. Nous avons discuté de l'ordre des énoncés du tableau de recommandations selon leur importance clinique et de la séquence dans laquelle les médecins auraient à les envisager pour leurs patients atteints de SLA.

Après l'obtention du soutien consensuel du groupe de travail à l'endroit des énoncés concernant les questions respiratoires, la Société canadienne de thoracologie (SCT) a publié une ligne directrice sur la ventilation mécanique à domicile pour les patients atteints de SLA ${ }^{35}$. Le groupe de travail a trouvé important que nos recommandations concordent avec cette ligne directrice. Nous avons donc comparé chacune des recommandations de la ligne directrice de la SCT à l'ébauche de nos énoncés. Notre groupe de travail a accepté la plupart des recommandations de la SCT sans modifications, si ce n'est certains choix de mots pour plus de cohérence. Nous avons demandé au comité sur la SLA de la SCT de revoir nos suggestions d'énoncés, y compris les énoncés consensuels des experts concernant les questions jugées importantes lors de notre sondage et qui n'étaient pas abordées dans la ligne directrice de la SCT; à la suite des commentaires reçus, des changements mineurs ont été apportés aux énoncés.

\section{Processus de révision}

Nous avons préparé un résumé des énoncés de la ligne directrice et le groupe de travail l'a révisé. Lorsque le groupe de travail a été satisfait du libellé des recommandations, y compris du choix de mots, de l'ordre et du classement des données probantes, nous l'avons envoyé aux membres du Réseau canadien de recherche sur la SLA (qui inclut toutes les cliniques multidisciplinaires de SLA au Canada) et à des experts externes (c.-à-d., gastro-entérologie, pneumologie, soins palliatifs et physiatrie) pour leurs commentaires. Nous avons aussi demandé aux cliniques de SLA de soumettre le résumé à leurs équipes multidisciplinaires pour qu'elles le commentent. Le groupe de travail a débattu de tous les commentaires reçus et déterminé quels changements s'imposaient, le cas échéant.

À partir du résumé révisé, une seconde revue externe a été effectuée par des intervenants clés de chaque société provinciale de SLA. Nous avons envoyé par courriel l'ébauche du résumé révisé accompagné d'un sondage comportant des questions ouvertes à chaque section provinciale de la SLA et nous avons demandé à chacune d'interroger ses membres, y compris 1 patient vivant avec la SLA. Tous les commentaires reçus ont été analysés individuellement par le groupe de travail qui y a apporté des modifications à sa discrétion après une discussion approfondie. Les changements consécutifs aux commentaires reçus concernaient surtout les choix de mots. Nous n'avons fait aucun changement substantiel.
Nous avons préparé une version intégrale de la ligne directrice que tous les membres du groupe de travail ont passée en revue pour approbation finale.

\section{Gestion des intérêts concurrents}

Tous les membres du groupe de travail ont agi sur une base volontaire et n'ont reçu aucune rémunération pour leur participation. La Société canadienne de la SLA et le Réseau canadien de recherche sur la SLA ont financé l'élaboration de la ligne directrice, y compris les déplacements pour les réunions en personne et la préparation du manuscrit en vue de sa publication.

La Société canadienne de la SLA est une organisation communautaire financée par des donateurs et une partie du financement de ce projet provient de dons amassés lors de l'événement Ice Bucket Challenge. La Société canadienne de la SLA a offert un soutien logistique, mais ne s'est pas prononcée sur le contenu des recommandations. Le Réseau canadien de recherche sur la SLA était un organisme à but non lucratif regroupant des médecins et des chercheurs qui se consacraient à la SLA; mis sur pied pour promouvoir la recherche clinique sur cette maladie au Canada, il était subventionné par des sociétés biotechnologiques qui soumettaient des propositions d'essais cliniques aux cliniques canadiennes de SLA. Le Réseau a par la suite fusionné avec la Société canadienne de la SLA. Même si des membres du Réseau ont participé à l'élaboration de la ligne directrice, le Réseau lui-même n'a joué aucun rôle dans son approbation.

Nous avons discuté de la gestion des intérêts concurrents durant la phase de planification de la ligne directrice; les intérêts concurrents ont été définis comme tout lien pécuniaire avec une société. À l'époque, il n'y avait qu'un seul médicament approuvé par Santé Canada pour le traitement de la SLA : le riluzole. Aucun des membres du groupe de travail ne présentait de conflits d'intérêts en lien avec le riluzole, qui est disponible depuis plus de 20 ans. Nous avons vérifié et confirmé qu'il n'y avait aucun autre conflit d'intérêts potentiel au sein du groupe de travail au début du projet.

En 2017, pendant le processus d'élaboration de la ligne directrice, la US Food and Drug Administration des États-Unis a approuvé l'édaravone pour traiter la SLA. Son fabricant, Mitsubishi Tanabe Pharma, a subventionné des comités consultatifs scientifiques sur l'utilisation de l'édaravone chez des patients atteints de SLA au Canada. En 2018, le médicament a été approuvé par Santé Canada et il a fait son entrée sur le marché canadien en novembre 2019. Certains membres du groupe de travail ont fait partie de comités consultatifs scientifiques de Mitsubishi Tanabe Pharma sur l'édaravone (C.S., M.C., A.I., W.J., C.O., K.S., L Z.); le groupe de travail en a été informé. Tous les membres du groupe de travail ont discuté longuement des énoncés de cette ligne directrice concernant l'édaravone. Pendant le processus de révision de l'ébauche de la ligne directrice, les commentaires des membres du Réseau canadien de recherche sur la SLA et des intervenants clés au sujet des énoncés concernant l'édaravone ont été traités par des membres du groupe de travail qui ne se trouvaient pas en situation de conflits d'intérêts avec Mitsubishi Tanabe Pharma (c.-à-d., n'ayant pas reçus d'honoraires de cette société). Les décisions finales concernant les 
énoncés sur l'édaravone ont été prises par des membres du groupe de travail qui n'étaient pas en situation potentielle de conflit d'intérêts. Outre l'édaravone, aucune situation potentielle de conflit d'intérêts n'a été soulevée en ce qui concerne les énoncés de cette ligne directrice.

\section{Mise en œuvre}

Les recommandations pour les pratiques optimales servent à guider les soins aux patients atteints de SLA au Canada. La ligne directrice sera publiée sur le site Web de la Société canadienne de la SLA (www.als.ca/bpr-appendix). La Société canadienne de la SLA participera aussi à la diffusion de la ligne directrice auprès des membres de la communauté concernée par la SLA, composée de médecins, de membres d'autres professions de la santé, de chercheurs, de patients et de leurs proches aidants, par l'entremise des sections provinciales de la SLA, du Réseau canadien de recherche sur la SLA et des participants au Forum annuel canadien de recherche sur la SLA. Les directeurs de cliniques de SLA et tous les médecins qui soignent des patients atteints de cette maladie seront encouragés à présenter la ligne directrice à leurs équipes cliniques et aux intervenants concernés dans leurs communautés respectives. La Société canadienne de la SLA soutiendra les auteurs de la ligne directrice en produisant des sommaires d'une page sur certains thèmes cliniques clés de la ligne directrice à l'intention des intervenants concernés.

Le groupe de travail appuierait un projet qui étudierait l'incidence de la ligne directrice sur la survie des patients, leur perception de leur qualité de vie et d'autres paramètres spécifiques après sa mise en œuvre comparativement à la période prépublication.

Le groupe de travail s'attend à ce que les données probantes concernant la prise en charge de la SLA évoluent au fil du temps et il prévoit devoir réviser les recommandations tous les 5 ans environ.

\section{Autres lignes directrices}

Plusieurs lignes directrices de pratique clinique pour la SLA ont été publiées ailleurs, notamment les paramètres de pratique de l'AAN (2009) $)^{6,7}$, la ligne directrice de la Fédération européenne des sociétés neurologiques sur la prise en charge clinique de la sclérose latérale amyotrophique $(2012)^{8}$ et la ligne directrice sur l'évaluation et la prise en charge de la maladie des motoneurones élaborée par le National Institute for Health and Care Excellence du Royaume-Uni (2016) .

L'un des objectifs de la ligne directrice canadienne était de mettre à jour les lignes directrices nord-américaines existantes, spécifiquement les recommandations de 2009 de l'AAN ${ }^{6,7}$. Comme on l'explique à la rubrique Méthodes, les interrogations de la littérature pour cette ligne directrice canadienne sur les questions cliniques abordées dans les recommandations de 2009 de l'AAN se sont limitées aux nouvelles données probantes seulement (c.-à-d., après 2007), qui ont toutes été classées selon les critères de l'AAN.
Dans la ligne directrice de l'AAN, les recommandations devaient s'appuyer sur des données probantes; ainsi, aucune orientation n'a été fournie en l'absence de données probantes (p. ex., par un consensus d'experts). En revanche, la ligne directrice de la Fédération européenne des sociétés neurologiques a formulé des recommandations à partir du consensus des experts en l'absence de données probantes. Et nous avons décidé de faire de même.

Un autre objectif de la ligne directrice canadienne était d'aborder des enjeux relatifs à la SLA qui étaient absents des autres lignes directrices. La ligne directrice de la Fédération européenne des sociétés neurologiques a ignoré plusieurs enjeux importants pour les patients atteints de SLA au Canada, tels que la gestion de la pharmacothérapie et l'AMM. De même, on ne trouvait rien sur certains enjeux touchant la SLA, tels que les traitements modificateurs de la maladie et l'exercice, dans la ligne directrice du National Institute for Health and Care Excellence.

Comme nous l'avons mentionné précédemment, la SCT a publié une ligne directrice sur la ventilation mécanique à domicile pour les patients atteints de SLA au début de 201935. En collaboration avec la SCT, nous avons veillé à ce que nos recommandations pour les soins respiratoires concordent avec celles de la SCT, mais nous avons ajouté certaines recommandations consensuelles (p. ex., sur la clairance des voies respiratoires).

\section{Connaissances à parfaire}

La ligne directrice confirme l'absence de données probantes de grande qualité pour la plupart des aspects de la prise en charge de la SLA; les recommandations formulées se fondent en général sur le consensus des experts du groupe de travail. Il faudra donc approfondir la recherche sur la prise en charge de la SLA, pour élaborer des recommandations fondées sur des données probantes si l'on veut améliorer les normes de soins au Canada et ailleurs. Cette ligne directrice peut orienter la communauté scientifique, ici et à l'étranger, vers les domaines de recherche clinique à prioriser pour la prise en charge de la maladie.

Nous reconnaissons que nous avons été incapables d'aborder tous les aspects de la prise en charge de la SLA dans la présente ligne directrice et que les révisions subséquentes pourraient inclure des aspects qui n'ont pas encore été touchés.

\section{Conclusion}

Nous espérons que l'élaboration de la première ligne directrice canadienne sur la SLA sera une importante avancée pour améliorer la vie des patients atteints de la maladie au Canada. Dans cette ligne directrice, la prépondérance des énoncés fondés sur le consensus des experts plutôt que sur des données probantes rappelle non seulement la nécessité d'approfondir la recherche sur la prise en charge de la SLA, mais souligne aussi les défis auxquels les médecins font face lorsqu'ils soignent des patients atteints de cette grave maladie invalidante. Cette ligne directrice permettra aux cliniques de SLA au Canada d'atteindre une norme nationale commune et de 
s'adapter à mesure que cette norme continuera d'évoluer. Ainsi, les médecins qui soignent des personnes atteintes de la SLA peuvent offrir des soins optimaux à leurs patients et les aider dans leur cheminement les confrontant à cette maladie extrêmement complexe et dévastatrice.

\section{Références}

1. Brown RH, Al-Chalabi A. Amyotrophic lateral sclerosis. N Engl J Med 2017;377: 162-72.

2. van Es MA, Hardiman $\mathrm{O}$, Chio A, et al. Amyotrophic lateral sclerosis. Lancet 2017;390:2084-98.

3. Strong MJ, Abrahams S, Goldstein LH, et al. Amyotrophic lateral sclerosis frontotemporal spectrum disorder (ALS-FTSD): Revised diagnostic criteria. Amyotroph Lateral Scler Frontotemporal Degener 2017;18:153-74.

4. Shoesmith C. A recipe for ALS. Can J Neurol Sci 2008;35:125-126.

5. Health Canada approves new drug to treat patients with amyotrophic lateral sclerosis (ALS) [communiqué de presse]. Ottawa : Health Canada; 2018 Oct. 4.

6. Miller RG, Jackson CE, Kasarskis EJ, et al. Practice parameter update: the care of the patient with amyotrophic lateral sclerosis: drug, nutritional, and respiratory therapies (an evidence-based review): report of the Quality Standards Subcommittee of the American Academy of Neurology. Neurology 2009;73: 1218-26.

7. Miller RG, Jackson CE, Kasarskis EJ, et al. Practice parameter update: the care of the patient with amyotrophic lateral sclerosis: multidisciplinary care, symptom management, and cognitive/behavioral impairment (an evidence-based review): report of the Quality Standards Subcommittee of the American Academy of Neurology. Neurology 2009;73:1227-33.

8. The EFNS Task Force on Diagnosis and Management of Amyotrophic Lateral Sclerosis, Andersen PM, Abrahams S, Borasio GD, et al. EFNS guidelines on the Clinical Management of Amyotrophic Lateral Sclerosis (MALS) - revised report of an EFNS task force. Eur J Neurol 2012;19:360-75.

9. Motor neurone disease: assessment and management (NG42). London (UK): National Institute for Healthcare and Excellence (NICE); 2016.

10. Peters M, Fitzpatrick R, Doll H, et al. Patients' experiences of health and social care in long-term neurological conditions in England: a cross-sectional survey. J Health Serv Res Policy 2013;18:28-33.

11. Abdulla S, Vielhaber S, Machts J, et al. Information needs and informationseeking preferences of ALS patients and their carers. Amyotroph Lateral Scler Frontotemporal Degener 2014;15:505-12.

12. Ang K, Umapathi T, Tong J, et al. Healthcare needs of patients with amyotrophic lateral sclerosis (ALS) in Singapore: a patient-centred qualitative study from multiple perspectives. J Palliat Care 2015;31:150-7.

13. Chiò A, Montuschi A, Cammarosano S, et al. ALS patients and caregivers communication preferences and information seeking behaviour. Eur J Neurol 2008; 15:55-60.

14. Miller RG, Mitchell JD, Moore DH. Riluzole for amyotrophic lateral sclerosis (ALS)/ motor neuron disease (MND). Cochrane Database Syst Rev 2012; (3):CD001447.

15. Rooney J, Byrne S, Heverin M, et al. Survival analysis of irish amyotrophic lateral sclerosis patients diagnosed from 1995-2010. PLoS One 2013;8:e74733.

16. Stevic Z, Kostic-Dedic S, Peric S, et al. Prognostic factors and survival of ALS patients from Belgrade, Serbia. Amyotroph Lateral Scler Frontotemporal Degener 2016;17:508-14.

17. Georgoulopoulou E, Fini N, Vinceti M, et al. The impact of clinical factors, riluzole and therapeutic interventions on ALS survival: a population-based study in Modena, Italy. Amyotroph Lateral Scler Frontotemporal Degener 2013;14:338-45.

18. Wei Q, Chen X, Zheng Z, et al. The predictors of survival in Chinese amyotrophic lateral sclerosis patients. Amyotroph Lateral Scler Frontotemporal Degener 2015;16:237-44.

19. Chen L, Liu X, Tang L, et al. Long-term use of riluzole could improve the prognosis of sporadic amyotrophic lateral sclerosis patients: a real-world cohort study in China. Front Aging Neurosci 2016;8:246.

20. Mandrioli J, Malerba SA, Beghi E, et al. Riluzole and other prognostic factors in ALS: a population-based registry study in Italy. J Neurol 2018;265:817-27.

21. Sívori M, Rodriguez GE, Pascansky D, et al. Outcome of sporadic amyotrophic lateral sclerosis treated with non-invasive ventilation and riluzole. Medicina ( $B$ Aires) 2007;67:326-30
22. Lee CT, Chiu YW, Wang KC, et al. Riluzole and prognostic factors in amyotrophic lateral sclerosis long-term and short-term survival: a population-based study of 1149 cases in Taiwan. J Epidemiol 2013;23:35-40.

23. Watanabe H, Atsuta N, Nakamura R, et al. Factors affecting longitudinal functional decline and survival in amyotrophic lateral sclerosis patients. Amyotroph Lateral Scler Frontotemporal Degener 2015;16:230-6.

24. Calvo A, Moglia C, Lunetta C, et al. Factors predicting survival in ALS: a multicenter Italian study. J Neurol 2017;264:54-63.

25. Keren N, Scott KM, Tsuda M, et al. Evidence of an environmental effect on survival in ALS. Amyotroph Lateral Scler Frontotemporal Degener 2014;15:528-33.

26. Fang T, Al Khleifat A, Meurgey JH, et al. Stage at which riluzole treatment prolongs survival in patients with amyotrophic lateral sclerosis: a retrospective analysis of data from a dose-ranging study. Lancet Neurol 2018;17:416-22.

27. Chen $X$, Wei QQ, Chen $Y$, et al. Clinical staging of amyotrophic lateral sclerosis in Chinese patients. Front Neurol 2018;9:442.

28. Zoccolella S, Beghi E, Palagano G, et al. Riluzole and amyotrophic lateral sclerosis survival: a population-based study in southern Italy. Eur J Neurol 2007;14:262-8.

29. Abe K, Itoyama Y, Sobue G, et al. Confirmatory double-blind, parallel-group, placebo-controlled study of efficacy and safety of edaravone ( $\mathrm{MCl}-186)$ in amyotrophic lateral sclerosis patients. Amyotroph Lateral Scler Frontotemporal Degener. 2014;15:610-7.

30. Writing Group, Edaravone ALSSG. Safety and efficacy of edaravone in well defined patients with amyotrophic lateral sclerosis: a randomised, doubleblind, placebo-controlled trial. Lancet Neurol. 2017;16:505-12.

31. Rooney J, Byrne S, Heverin M, et al. A multidisciplinary clinic approach improves survival in ALS: a comparative study of ALS in Ireland and Northern Ireland. J Neurol Neurosurg Psychiatry 2015;86:496-501.

32. Traynor BJ, Alexander M, Corr B, et al. Effect of a multidisciplinary amyotrophic lateral sclerosis (ALS) clinic on ALS survival: a population-based study, 1996-2000. $J$ Neurol Neurosurg Psychiatry 2003;74:1258-61.

33. Van den Berg JP, Kalmijn S, Lindeman E, et al. Multidisciplinary ALS care improves quality of life in patients with ALS. Neurology 2005;65:1264-7.

34. Geronimo A, Wright C, Morris A, et al. Incorporation of telehealth into a multidisciplinary ALS Clinic: feasibility and acceptability. Amyotroph Lateral Scler Frontotemporal Degener 2017;18:555-61.

35. Rimmer KP, Kaminska M, Nonoyama M, et al. Home mechanical ventilation for patients with amyotrophic lateral sclerosis: a Canadian Thoracic Society clinical practice guideline. Can J Respir Cri Care Sleep Med 2019;3:9-27.

36. Gonzalez Calzada N, Prats Soro E, Mateu Gomez L, et al. Factors predicting survival in amyotrophic lateral sclerosis patients on non-invasive ventilation. Amyotroph Lateral Scler Frontotemporal Degener 2016;17:337-42.

37. Silva LB, Mourao LF, Silva AA, et al. Effect of nutritional supplementation with milk whey proteins in amyotrophic lateral sclerosis patients. Arq Neuropsiquiatr 2010;68:263-8.

38. Dorst J, Cypionka J, Ludolph AC. High-caloric food supplements in the treatment of amyotrophic lateral sclerosis: a prospective interventional study. Amyotroph Lateral Scler Frontotemporal Degener 2013;14:533-6.

39. Wills AM, Hubbard J, Macklin EA, et al. Hypercaloric enteral nutrition in patients with amyotrophic lateral sclerosis: a randomised, double-blind, placebo-controlled phase 2 trial. Lancet 2014;383:2065-72.

40. Gladman M, Dehaan M, Pinto $\mathrm{H}$, et al. Venous thromboembolism in amyotrophic lateral sclerosis: a prospective study. Neurology 2014;82:1674-7.

41. Qureshi MM, Cudkowicz ME, Zhang $H$, et al. Increased incidence of deep venous thrombosis in ALS. Neurology 2007;68:76-7.

42. Jackson CE, Gronseth G, Rosenfeld J, et al. Randomized double-blind study of botulinum toxin type B for sialorrhea in ALS patients. Muscle Nerve 2009;39:137-43.

43. Brooks BR, Thisted RA, Appel SH, et al. Treatment of pseudobulbar affect in ALS with dextromethorphan/quinidine: a randomized trial. Neurology 2004;63:1364-70.

44. Caron J, Light J. "My world has expanded even though i'm stuck at home": experiences of individuals with amyotrophic lateral sclerosis who use augmentative and alternative communication and social media. Am J Speech Lang Pathol 2015;24:680-95

45. Hwang C-S, Weng $\mathrm{H}-\mathrm{H}$, Wang L-F, et al. An eye-tracking assistive device improves the quality of life for ALS patients and reduces the caregivers' burden. J Mot Behav 2014;46:233-8.

46. Clawson LL, Cudkowicz M, Krivickas L, et al. A randomized controlled trial of resistance and endurance exercise in amyotrophic lateral sclerosis. Amyotroph Lateral Scler Frontotemporal Degener 2018;19:250-8. 
47. Lunetta C, Lizio A, Sansone VA, et al. Strictly monitored exercise programs reduce motor deterioration in ALS: preliminary results of a randomized controlled trial. J Neurol 2016;263:52-60.

48. Elamin M, Phukan J, Bede P, et al. Executive dysfunction is a negative prognostic indicator in patients with ALS without dementia. Neurology 2011;76:1263-9.

49. Burke T, Hardiman O, Pinto-Grau M, et al. Longitudinal predictors of caregiver burden in amyotrophic lateral sclerosis: a population-based cohort of patientcaregiver dyads. J Neurol 2018;265:793-808.

50. Lerum SV, Solbraekke KN, Frich JC. Family caregivers' accounts of caring for a family member with motor neurone disease in Norway: a qualitative study. BMC Palliat Care 2016;15:22.

51. Watermeyer TJ, Brown RG, Sidle KC, et al. Impact of disease, cognitive and behavioural factors on caregiver outcome in amyotrophic lateral sclerosis. Amyotroph Lateral Scler Frontotemporal Degener 2015;16:316-23.

52. Foley G, Timonen V, Hardiman O. "I hate being a burden": the patient perspective on carer burden in amyotrophic lateral sclerosis. Amyotroph Lateral Scler Frontotemporal Degener 2016;17:351-7.

53. Mitsumoto $\mathrm{H}$, Bromberg $\mathrm{M}$, Johnston $\mathrm{W}$, et al. Promoting excellence in end-of-life care in ALS. Amyotroph Lateral Scler Other Motor Neuron Disord 2005;6:145-54.
54. Ray RA, Brown J, Street AF. Dying with motor neurone disease, what can we learn from family caregivers? Health Expect 2014;17:466-76.

55. Lulé D, Nonnenmacher S, Sorg S, et al. Live and let die: existential decision processes in a fatal disease. J Neurol 2014;261:518-25.

56. Murray L, Butow PN, White K, et al. Advance care planning in motor neuron disease: A qualitative study of caregiver perspectives. Palliat Med 2016;30:471-8.

57. Burchardi N, Rauprich O, Hecht M, et al. Discussing living wills. A qualitative study of a German sample of neurologists and ALS patients. J Neurol Sci 2005; 237:67-74.

58. Preston H, Fineberg IC, Callagher $\mathrm{P}$, et al. The preferred priorities for care document in motor neurone disease: views of bereaved relatives and carers. Palliat Med 2012;26:132-8.

59. Sulmasy DP, Terry PB, Weisman CS, et al. The accuracy of substituted judgments in patients with terminal diagnoses. Ann Intern Med 1998;128:621-9.

60. Schünemann HJ, Wiercioch W, Etxeandia I, et al. Guidelines 2.0: systematic development of a comprehensive checklist for a successful guideline enterprise. CMAJ 2014;186:E123-42.

61. Clinical practice guideline process manual. St. Paul (MN): AAN (American Academy of Neurology); 2011.
Intérêts concurrents : Christen Shoesmith, Aaron Izenberg, Wendy Johnston, Colleen O'Connell, Kerri Schellenberg et Lorne Zinman ont été membres d'un comité consultatif scientifique sur Radicava (édaravone; Mitsubishi Tanabe Pharma Canada). Christen Shoesmith signale avoir été investigatrice principale d'un site pour plusieurs essais cliniques multicentriques sur la sclérose latérale amyotrophique (SLA). Au cours des 36 derniers mois, la $D^{\text {re }}$ Shoesmith a participé à des essais cliniques commandités par Biogen, Cytokinetics, ALS Pharma et Orphazyme. Marvin Chum signale avoir reçu une bourse de recherche clinique sur la SLA du programme Bernice Ramsay, indépendamment des travaux soumis. Aaron Izenberg signale avoir reçu des honoraires personnels de Biogen, Roche, Alnylam, Genzyme, Takeda et Mitsubishi Tanabe Pharma, indépendamment des travaux soumis. Aucun autre intérêt concurrent n'a été déclaré.

Cet article a été révisé par des pairs.

Affiliations : Centre des sciences de la santé de London (Shoesmith), London, Ont.; Centre des sciences de la santé Sunnybrook (Abrahao, Izenberg, Tandon, Zinman), Toronto, Ont.; Université Dalhousie (Benstead, Leddin), Halifax, N.-É.; Université McMaster (CHUM), Hamilton, Ont.; CHU de Québec-Université Laval (Dupré), Québec, Qué.; Université de l'Alberta (Johnston, Kalra), Edmonton, Alta.; Centre de réadaptation Stan Cassidy (O'Connell), Fredericton, N.-B.; Université de la Saskatchewan (Schellenberg), Saskatoon, Sask.
Collaborateurs : Tous les auteurs ont contribué à l'élaboration et à la conception des travaux ainsi qu'à l'acquisition, à l'analyse et à l'interprétation des données. Tous les auteurs ont participé à la rédaction du manuscrit, en ont révisé de façon critique le contenu intellectuel important, ont donné leur approbation finale pour la version destinée à être publiée, et assument l'entière responsabilité de tous les aspects du travail.

Financement : Le financement pour l'élaboration des recommandations a été fourni par la Société canadienne de la SLA et le Réseau canadien de recherche sur la SLA.

Remerciements : L'équipe de rédaction remercie la Société canadienne de la SLA pour son soutien continu à l'élaboration de cette ligne directrice. Les auteurs remercient aussi $\mathrm{M}^{\mathrm{me}}$ Vanessa Blount, qui a aidé à la coordination initiale de la ligne directrice, M. David Taylor et $\mathrm{M}^{\mathrm{me}}$ Colleen Doyle pour leur soutien logistique, et $\mathrm{M}^{\mathrm{me}}$ Trisha Rao, qui a collaboré à la révision du document. Le Réseau canadien de recherche sur la SLA et des partenaires de la fédération ont aussi formulé des commentaires critiques en ce qui concerne le libellé des recommandations et du manuscrit. Les $D^{\text {rs }}$ Ikhlass Salem Haj, Lawrence Korngut et Hannah Briemberg ont eux aussi fourni une aide substantielle à l'élaboration de ces recommandations.

Correspondance : Christen Shoesmith, Christen.Shoesmith@lhsc.on.ca 